Pacific

Journal of

Mathematics

\title{
FREE BROWNIAN MOTION AND FREE CONVOLUTION SEMIGROUPS: MULTIPLICATIVE CASE
}

PING ZHONG 


\title{
FREE BROWNIAN MOTION AND FREE CONVOLUTION SEMIGROUPS: MULTIPLICATIVE CASE
}

\author{
PING ZHONG
}

\begin{abstract}
We consider a pair of probability measures $\mu$, $v$ on the unit circle such that $\Sigma_{\lambda}\left(\eta_{v}(z)\right)=z / \eta_{\mu}(z)$. We prove that the same type of equation holds for any $t \geq 0$ when we replace $v$ by $v \otimes \lambda_{t}$ and $\mu$ by $\mathbb{M}_{t}(\mu)$, where $\lambda_{t}$ is the free multiplicative analogue of the normal distribution on the unit circle of $\mathbb{C}$ and $\mathbb{M}_{t}$ is the map defined by Arizmendi and Hasebe. These equations are a multiplicative analogue of equations studied by Belinschi and Nica. In order to achieve this result, we study infinite divisibility of the measures associated with subordination functions in multiplicative free Brownian motion and multiplicative free convolution semigroups. We use the modified $\mathscr{S}$-transform introduced by Raj Rao and Speicher to deal with the case that $v$ has mean zero. The same type of the result holds for convolutions on the positive real line. In the end, we give a new proof for some Biane's results on the densities of the free multiplicative analogue of the normal distributions.
\end{abstract}

\section{Introduction}

Let $\mathcal{M}_{\mathbb{R}}$ be the set of probability measures on $\mathbb{R}$. For every $t \geq 0$, Belinschi and Nica [2008b] defined a family of maps $\mathbb{B}_{t}: \mathcal{M}_{\mathbb{R}} \rightarrow \mathcal{M}_{\mathbb{R}}$ by setting

$$
\mathbb{B}_{t}(\mu)=\left(\mu^{\boxplus(t+1)}\right)^{\uplus 1 /(t+1)}, \quad \mu \in \mathcal{M}_{\mathbb{R}} .
$$

These maps have several remarkable properties. For any $t \geq 0, \mathbb{B}_{t}$ is an endomorphism of $\left(\mathcal{M}_{\mathbb{R}^{+}}, \bigotimes\right)$, where $\mathcal{M}_{\mathbb{R}^{+}}$is the set of probability measures on $[0,+\infty)$ and $\triangle$ is free multiplicative convolution. $\left\{\mathbb{B}_{t}\right\}_{t \geq 0}$ is a semigroup and $\mathbb{B}_{1}$ is the Boolean to free Bercovici-Pata bijective map.

The maps $\mathbb{B}_{t}$ have strong connections with $\boxplus$-infinite divisibility. They are also connected to free Brownian motion and additive free convolution semigroups. For $\mu \in \mathcal{M}_{\mathbb{R}}$, we denote by $G_{\mu}$ the Cauchy transform of $\mu$ and by $F_{\mu}$ the reciprocal Cauchy transform of $\mu$. Given a pair of probability measures $\mu, v \in \mathcal{M}_{\mathbb{R}}$ such that

$$
G_{v}(z)=z-F_{\mu}(z), \quad z \in \mathbb{C}^{+},
$$

MSC2010: 46L54.

Keywords: multiplicative free convolution, free Brownian motion, free convolution semigroups. 
we have

$$
G_{\nu \boxplus \gamma_{t}}(z)=z-F_{\mathbb{B}_{t}(\mu)}(z), \quad t>0, z \in \mathbb{C}^{+},
$$

where $\gamma_{t}$ is the semicircular distribution with variance $t$. This result was generalized to the multi-variable case in [Belinschi and Nica 2008a; 2009; Nica 2009]. An equivalent form of (1-1) was used to prove the superconvergence theorem in [Wang 2010]. Anshelevich [2010; 2011a; 2011b; 2012] generalized the above correspondence of $\mu \leftrightarrow \nu$ and $\mathbb{B}_{t}(\mu) \leftrightarrow v \boxplus \gamma_{t}$ to the context of two-state probability spaces. Motivated by these generalizations and applications, we study in this article the analogue of these equations for multiplicative free convolution.

Throughout this article, we denote by $\mathrm{T}$ the unit circle of $\mathbb{C}$, by $\mathcal{M}_{\mathrm{T}}$ the set of probability measures on $\mathrm{T}$, and by $M_{*}$ the set of probability measures on $\mathbb{C}$ with nonzero mean. We also set

$$
\mathcal{M}_{\mathrm{T}}^{*}=\left\{\mu \in \mathcal{M}_{\mathrm{T}} \cap \mathcal{M}_{*}: \eta_{\mu}(z) \neq 0 \text { for all } z \in \mathbb{D} \backslash\{0\}\right\} .
$$

It was shown in [Belinschi and Bercovici 2005] that one can define multiplicative free convolution power $\mu^{\otimes t}$ for $\mu \in M_{\mathrm{T}}^{*}$ and $t>1$.

In [Arizmendi and Hasebe 2013], a family of maps $\mathbb{M}_{t}$, which is the analogue of the semigroup $\mathbb{B}_{t}$, was defined for the probability measures in $M_{\mathrm{T}}^{*}$. The definition of $\mathbb{M}_{t}$ there was more general; we only need a simpler form defined as follows. Given $\mu \in M_{\mathrm{T}}^{*}$ having positive mean, then for $t \geq 0$, the map $\mathbb{M}_{t}$ is defined by

$$
\mathbb{M}_{t}(\mu)=\left(\mu^{\otimes(t+1)}\right)^{凶 1 /(t+1)},
$$

where the convolution power $\mu^{\bigotimes(t+1)}$ and the measure $\mathbb{M}_{t}(\mu)$ are chosen in a way such that they have positive means.

We then state one of our main theorems.

Theorem 1.1. Given a pair of probability measures $\mu \in M_{\mathrm{T}}^{*}$ and $v \in M_{\mathrm{T}}$ such that

$$
\Sigma_{\lambda}\left(\eta_{\nu}(z)\right)=\frac{z}{\eta_{\mu}(z)}, \quad z \in \mathbb{D},
$$

we have

$$
\Sigma_{\lambda}\left(\eta_{\nu \otimes \lambda_{t}}(z)\right)=\frac{z}{\eta_{\mathbb{M}_{t}(\mu)}(z)}, \quad z \in \mathbb{D},
$$

where $\lambda_{t}$ is the analogue of the normal distribution on $T$ with $\Sigma_{\lambda_{t}}(z)=\exp ((t / 2)(1+$ $z) /(1-z))$ and $\lambda=\lambda_{1}$.

In order to prove Theorem 1.1, we consider two semigroups $\nu \otimes \lambda_{t}$ and $\mu^{\bigotimes(t+1)}$ for all $t \geq 0$. It is well-known that $\eta_{\nu \otimes \lambda_{t}}$ and $\eta_{\mu} \otimes(t+1)$ are subordinated to $\eta_{\nu}$ and $\eta_{\mu}$ respectively. We prove that the subordination functions are $\eta$-transforms of some $\triangle$-infinitely divisible probability measures on $T$. It turns out that the equation 
$\Sigma_{\lambda}\left(\eta_{\nu}(z)\right)=z / \eta_{\mu}(z)$ means that the subordination function of $\eta_{\nu \otimes \lambda_{t}}$ with respect to $\eta_{\nu}$ and the subordination function of $\eta_{\mu} \bowtie(t+1)$ with respect to $\eta_{\mu}$ are the same. The proof of Theorem 1.1 will be given in Subsection 3.5.

Given $\mu \in M_{\mathrm{T}}$, we prove that if $\mu^{\bigotimes t}$ can be defined and $\eta_{\mu}{ }_{t}$ is subordinated to $\eta_{\mu}$ for all $t>1$, then $\mu \in M_{\mathrm{T}}^{*}$; in addition, we prove that for nontrivial measures $\mu \in \mathcal{M}_{\mathrm{T}}$ and $v \in \mathscr{I} \mathscr{D}(\bigotimes, \mathrm{T})$, the density functions of the measures $\mu \otimes v_{t}$ and $\mu^{\bigotimes t}$ converge to $1 / 2 \pi$ uniformly as $t \rightarrow \infty$.

To deal with the case that $v \in M_{T} \backslash M_{*}$, we use the modified $\mathscr{Y}$-transform [Arizmendi 2012; Rao and Speicher 2007] to study subordination functions. In this case, the subordination function of $\eta_{\nu \otimes \lambda_{t}}$ with respect to $\eta_{\nu}$ is generally not unique. However, we can prove that there exists a unique subordination function satisfying certain properties (see Theorem 3.11). Let $\rho_{t}$ be the measure associated with this subordination function of $\eta_{\nu \bowtie \lambda_{t}}$ with respect to $\eta_{\nu}$, we have that $\Sigma_{\rho_{t}}(z)=\Sigma_{\lambda_{t}}\left(\eta_{\nu}(z)\right)$.

Similar results to Theorem 1.1 for multiplicative convolution on $M_{\mathbb{B}^{+}}$are also valid. The proof for this case is much simpler because of the uniqueness of multiplicative convolution powers and the uniqueness of subordination functions.

Finally, we give a new proof for some results concerning the density functions of the free multiplicative analogue of the normal distributions studied by Biane [1997c], and we obtain some new results. For example, for $\lambda_{t}(t>0)$ the free multiplicative analogue of the normal distributions on $\mathrm{T}$, we prove that $\lambda_{t}$ is unimodal.

This article is organized as follows. After this introductory section, we describe some backgrounds in the additive case in Section 2. In Section 3, we consider multiplicative free and multiplicative Boolean convolution on $\mu_{\mathrm{T}}$, and prove our main theorems. Section 4 is devoted to studying multiplicative free and multiplicative Boolean convolution on $\mathcal{M}_{\mathbb{R}^{+}}$. The regularity properties of the free multiplicative analogue of the normal distributions are discussed in Section 5.

\section{Background: additive case}

Additive free convolution and additive Boolean convolution. For a measure $\mu \in$ $\mathcal{M}_{\mathbb{R}}$, we define the Cauchy transform $G_{\mu}: \mathbb{C}^{+} \rightarrow \mathbb{C}^{-}$by

$$
G_{\mu}(z)=\int_{-\infty}^{+\infty} \frac{1}{z-t} d \mu(t), \quad z \in \mathbb{C}^{+}
$$

We set

$$
F_{\mu}(z)=\frac{1}{g \mu(z)}, \quad z \in \mathbb{C}^{+},
$$

so that $F_{\mu}: \mathbb{C}^{+} \rightarrow \mathbb{C}^{+}$is analytic.

The following result characterizes those functions which are reciprocal Cauchy transforms of probability measures. 
Proposition 2.1 [Bercovici and Voiculescu 1993]. Let $F: \mathbb{C}^{+} \rightarrow \mathbb{C}^{+}$be an analytic function. The following assertions are equivalent:

(1) There exists a probability measure $\mu$ on $\mathbb{R}$ such that $F(z)=F_{\mu}(z)$ in $\mathbb{C}^{+}$.

(2) There exists $a \in \mathbb{R}$, and a finite positive measure $\rho$ on $\mathbb{R}$ such that

$$
F(z)=a+z+\int_{-\infty}^{+\infty} \frac{1+t z}{t-z} d \rho(t) \quad \text { for all } z \in \mathbb{C}^{+} .
$$

(3) $\lim _{y \rightarrow+\infty} F(i y) / i y=1$.

It was proved in [Bercovici and Voiculescu 1993] that $F_{\mu}$ is invertible in some domain. More precisely, for two positive numbers $M$ and $N$, we set

$$
\Gamma_{M, N}=\left\{z \in \mathbb{C}^{+}:|x|<M y, y>N\right\} .
$$

Then for any $M>0$, there exists $N>0$ such that the left inverse $F_{\mu}^{-1}$ of $F_{\mu}$ is defined in $\Gamma_{M, N}$, and then we can define the Voiculescu transform of $\mu$ by

$$
\varphi_{\mu}(z)=F_{\mu}^{-1}(z)-z
$$

for $z \in \Gamma_{M, N}$. For any two measures $\mu, v \in \mathcal{M}_{\mathbb{R}}$, we have

$$
\varphi_{\mu \boxplus v}(z)=\varphi_{\mu}(z)+\varphi_{\nu}(z)
$$

in any truncated cone $\Gamma_{M, N}$ where $\varphi_{\mu}, \varphi_{\nu}$ and $\varphi_{\mu \boxplus v}$ are defined. This remarkable result was proved in [Voiculescu 1986] for compactly supported measures and then extended to general cases in [Bercovici and Voiculescu 1993; Maassen 1992].

Given $v \in \mathcal{M}_{\mathbb{R}}$, we say that $v$ is $\boxplus$-infinitely divisible if for every positive integer $n$, there exists a probability measure $v_{1 / n} \in \mathcal{M}_{\mathbb{R}}$ such that

$$
v=\underbrace{v_{1 / n} \boxplus v_{1 / n} \boxplus \cdots \boxplus v_{1 / n}}_{n \text { times }} .
$$

It is known [Bercovici and Voiculescu 1993; Maassen 1992; Voiculescu 1986] that a probability measure $v$ on $\mathbb{R}$ is $\boxplus$-infinitely divisible if and only if its Voiculescu transform $\varphi_{v}$ has an analytic extension defined on $\mathbb{C}^{+}$with values in $\mathbb{C}^{-} \cup \mathbb{R}$. We denote by $\mathscr{\mathscr { D }}(\boxplus, \mathbb{R})$ the set of all $\boxplus$-infinitely divisible probability measures on the real line. If $v \in \mathscr{I}(\boxplus, \mathbb{R})$, then for every $t>0$, there exists a probability measure $v_{t}$ such that $\varphi_{v_{t}}(z)=t \varphi_{v}(z)$ for $z$ in the common domain of $\varphi_{v}$ and $\varphi_{v_{t}}$.

Proposition 2.2. Let $v$ is $\boxplus$-infinitely divisible, and let $H(z)=z+\varphi_{v}(z)$. Then

$$
H\left(F_{v}(z)\right)=z
$$

for $z \in \mathbb{C}^{+}$. The set $U:=\left\{z \in \mathbb{C}^{+}: \Im H(z)>0\right\}$ is a simply connected domain with boundary which is a simple curve and $H$ maps $\mathbb{C}^{+}$conformally onto $U$. Moreover, 
the boundary $\partial U$ is the graph of a function and the function $H$ is continuous up to the real axis.

Proof. The first part of the assertion appears in [Bercovici and Voiculescu 1993; Voiculescu 1986], and the second part of the assertion follows from the fact that $H$ satisfies the conditions of [Belinschi and Bercovici 2005, Proposition 4.7]. The last part of the assertion is due to [Chistyakov and Götze 2013, Lemma 3.3; Belinschi and Bercovici 2005, Proposition 4.7].

Additive Boolean convolution was introduced in [Speicher and Woroudi 1997]. For $\mu \in \mathcal{M}_{\mathbb{R}}$, we set $E_{\mu}(z)=z-F_{\mu}(z)$. For $\mu, v \in \mathcal{M}_{\mathbb{R}}$, the additive Boolean convolution $\mu \uplus v$ is characterized by the identity

$$
E_{\rho}(z)=E_{\mu}(z)+E_{v}(z) \text { for } z \in \mathbb{C}^{+} .
$$

We can also consider the infinite divisibility with respect to additive Boolean convolution. It turns out that every $\mu \in \mathcal{M}_{\mathbb{R}}$ is $\uplus$-infinitely divisible; see [Speicher and Woroudi 1997]. We denote by $\mathscr{I} \mathscr{D}(\uplus, \mathbb{R})$ the set of all $\uplus$-infinitely divisible probability measures on the real line.

Infinite divisibility and subordination functions. Given $\mu, v \in M_{\mathbb{R}}$, it is known that $F_{\mu \boxplus \nu}$ is subordinated to $F_{\mu}$ and $F_{\nu}$, and by Proposition 2.1, we can also regard these subordination functions as the reciprocal Cauchy transforms of probability measures on $\mathbb{R}$.

Definition 2.3. For $\mu, v \in M_{\mathbb{R}}$, the subordination distribution [Anshelevich 2012; Lenczewski 2007; Nica 2009] $\mu \boxminus v$ is defined to be the unique probability measure in $\mathcal{M}_{\mathbb{R}}$ such that $F_{\mu \boxplus v}(z)=F_{\nu}\left(F_{\mu \boxplus v}(z)\right)$.

Many subordination distributions in semigroups related to free convolution are infinitely divisible; see [Anshelevich 2012; Nica 2009].

Proposition 2.4. Let $\mu, v \in \mathcal{M}_{\mathbb{R}}$.

(1) $\varphi_{\mu \boxplus v}(z)=\left(\varphi_{\mu} \circ F_{v}\right)(z)$.

(2) If $\mu \in \mathscr{I} \mathscr{D}(\boxplus, \mathbb{R})$, then $\mu \boxminus v \in \mathscr{I}(\boxplus, \mathbb{R})$. In particular, $\gamma_{t} \boxplus v \in \mathscr{I}(\boxplus, \mathbb{R})$ and $\varphi_{\gamma_{t} \boxplus v}(z)=t G_{v}(z)$, where $\gamma_{t}$ is the semicircular distribution with variance $t$.

(3) If $v=\mu \boxplus v^{\prime}$ for $v^{\prime} \in \mathcal{M}_{\mathbb{R}}$, then $\mu \boxplus v \in \mathscr{D}(\boxplus$, $\mathbb{R})$. In particular, $\mu \boxplus \mu \in$ $\mathscr{S}(\boxplus, \mathbb{R})$, and $\varphi_{\mu \boxplus \mu}(z)=z-F_{\mu}(z)$.

Proof. Part (1) is Lemma 1 of [Anshelevich 2012]. Note that $\varphi_{\gamma_{t}}(z)=t / z$ and $\left(\varphi_{\mu} \circ F_{\mu}\right)(z)=z-F_{\mu}(z)$; parts (2) and (3) follow from part 1 and Lemma 2 of [Anshelevich 2012]. See also [Chistyakov and Götze 2011, Corollary 2.3].

The following result was inspired by a question of Michael Anshelevich [2012], to whom I am grateful for sending me an updated version of his paper. 
Lemma 2.5. Given $\tau, \rho \in \mathcal{M}_{\mathbb{R}}$, if $\tau \boxplus \rho \in \mathscr{I}(\boxplus, \mathbb{R})$, then $\rho \boxplus \tau^{\boxplus t}$ is defined for all $t \geq 0$ in the sense that $\varphi_{\rho}+t \varphi_{\tau}$ is the Voiculescu transform of a positive measure. Moreover, $F_{\rho \boxplus\left(\tau^{\boxplus t}\right)}=F_{\rho}\left(F_{(\tau \boxplus \rho)^{\boxplus t}}(z)\right)$.

Proof. Let $\sigma=\tau \boxplus \rho$ and $\sigma_{t}=\sigma^{\boxplus t}$. By Proposition 2.1, there exists a unique probability measure $\mu_{t} \in \mathcal{M}_{\mathbb{R}}$ such that

$$
F_{\mu_{t}}=F_{\rho}\left(F_{\sigma_{t}}(z)\right) .
$$

We claim that $\varphi_{\mu_{t}}(z)=\varphi_{\rho}(z)+t \varphi_{\tau}(z)$. Indeed, by Proposition 2.4, we have

$$
F_{\sigma_{t}}^{-1}(z)-z=t \cdot \varphi_{\sigma}(z)=t \cdot \varphi_{\tau}\left(F_{\rho}(z)\right),
$$

and we thus obtain

$$
\begin{aligned}
\varphi_{\mu_{t}}\left(F_{\rho}(z)\right) & =F_{\mu_{t}}^{-1}\left(F_{\rho}(z)\right)-F_{\rho}(z)=F_{\sigma_{t}}^{-1}(z)-F_{\rho}(z)=F_{\sigma_{t}}^{-1}(z)-z+z-F_{\rho}(z) \\
& =t \cdot \varphi_{\tau}\left(F_{\rho}(z)\right)+F_{\rho}^{-1}\left(F_{\rho}(z)\right)-F_{\rho}(z) .
\end{aligned}
$$

By analytic continuation, we conclude that

$$
\varphi_{\mu_{t}}(z)=t \cdot \varphi_{\tau}(z)+F_{\rho}^{-1}(z)-z=\varphi_{\rho}(z)+t \cdot \varphi_{\tau}(z),
$$

which completes the proof.

Remark. There are examples $\rho, \tau \in \mathcal{M}_{\mathbb{R}}$ such that $\tau \boxminus \rho \in \mathscr{I}(\boxplus$, $\mathbb{R})$ but $\tau$ does not lie in $\mathscr{\mathscr { D }}(\boxplus, \mathbb{R})$ and is not a summand of $\rho$; see [Anshelevich 2012].

Combining Proposition 2.4 and Lemma 2.5, we can reconstruct Nica-Speicher free convolution semigroups [Belinschi and Bercovici 2004; Nica and Speicher 1996] as follows.

Theorem 2.6. Given $\mu \in \mathcal{M}_{\mathbb{R}}$, the measure $\mu^{\boxplus t} \in \mathcal{M}_{\mathbb{R}}$ is defined by $\varphi_{\mu^{\boxplus t}}(z)=t \varphi_{\mu}(z)$ for all $t>1$. Moreover, there exists an analytic map $\omega_{t}: \mathbb{C}^{+} \rightarrow \mathbb{C}^{+}$such that for $z \in \mathbb{C}^{+}$the following conditions are satisfied:

- $F_{\mu} \boxplus t(z)=F_{\mu}\left(\omega_{t}(z)\right)$.

- $\omega_{t}=F_{(\mu \boxplus \mu)^{\boxplus(t-1)}}(z)$.

- $\varphi_{(\mu \boxplus \mu)^{\boxplus(t-1)}}=(t-1)\left(z-F_{\mu}(z)\right)$ for all $t>1$.

Let $H_{t}(z)=z+(t-1)\left(z-F_{\mu}(z)\right)$. By Proposition 2.2 and Theorem 2.6, we know that $H_{t}$ is the left inverse of $\omega_{t}$ such that $H_{t}\left(\omega_{t}(z)\right)=z$ for $z \in \mathbb{C}^{+}$. Therefore, for $t>1$, we can write

$$
\omega_{t}(z)=z+\left(1-\frac{1}{t}\right)\left(F_{\mu^{\boxplus t}}(z)-z\right), \quad z \in \mathbb{C}^{+} .
$$

We deduce from (2-3) and the definition of $\omega_{t}$ in Theorem 2.6 that, for $t>0$,

$$
z-F_{(\mu \boxplus \mu)^{\boxplus t}}(z)=\left(1-\frac{1}{t+1}\right)\left(z-F_{\mu^{\boxplus(t+1)}}(z)\right),
$$


which implies that

$$
(\mu \boxminus \mu)^{\boxplus t}(z)=\left(\mu^{\boxplus(t+1)}\right)^{\uplus(t /(t+1))} .
$$

Two formulas related to free Brownian motion. Given $\mu \in M_{\mathbb{R}}$, we construct subordination functions $\omega_{t}$ as in Theorem 2.6. Let $\sigma_{t}=(\mu \boxminus \mu)^{\boxplus t} \in \mathcal{M}_{\mathbb{R}}$; then $\omega_{t+1}=F_{\sigma_{t}}(z)$ for $t>0$. Given $v \in \mathcal{M}_{\mathbb{R}}$, let $\rho_{t}=\gamma_{t} \boxplus v$ and let $F_{t}=F_{\rho_{t}}(z)$ for all $t>0$. From Proposition 2.4 and Theorem 2.6, we know that $\rho_{t}$ and $\sigma_{t}$ are $\boxplus$-infinitely divisible and their Voiculescu transforms are given by $\varphi_{\rho_{t}}(z)=t G_{\nu}$ and $\varphi_{\sigma_{t}}(z)=t\left(z-F_{\mu}(z)\right)$. By comparing Voiculescu transform of $\rho_{t}$ with Voiculescu transform of $\sigma_{t}$, we deduce that $F_{t}=\omega_{t+1}$ for some $t>0$ if and only if $G_{v}(z)=$ $z-F_{\mu}(z)$.

For $t>0$, Belinschi and Nica [2008b] constructed a transformation $\mathbb{B}_{t}: M_{\mathbb{R}} \rightarrow M_{\mathbb{R}}$ such that

$$
\mathbb{B}_{t}(\mu)=\left(\mu^{\boxplus(1+t)}\right)^{\uplus 1 /(1+t)} \quad \text { for } \mu \in \mathcal{M}_{\mathbb{B}} .
$$

They also showed that $\mathbb{B}_{t}$ is a semigroup and $\mathbb{B}_{2}=\mathbb{B}$, where

$$
\mathbb{B}: \mathscr{I}(\uplus, \mathbb{R}) \rightarrow \mathscr{I}(\boxplus, \mathbb{R})
$$

is the bijective map from the $\uplus$-infinitely divisible distributions to the $\boxplus$-infinitely divisible distributions, discovered in the seminal [Bercovici and Pata 1999].

Theorem 2.7 [Belinschi and Nica 2008b]. Let $\mu$ and $v$ be a pair of probability measures on the real line such that

$$
G_{v}(z)=z-F_{\mu}(z), \quad z \in \mathbb{C}^{+} .
$$

Then we have

$$
G_{\nu \boxplus \gamma_{t}}(z)=z-F_{\mathbb{B}_{t}(\mu)}(z), \quad t>0, z \in \mathbb{C}^{+} .
$$

Remark. Maassen [1992] has shown that, given $\mu, v \in \mathcal{M}_{\mathbb{R}}$ satisfying (2-5), $\mu$ has mean zero and variance one. Conversely, if $\mu \in \mathcal{M}_{\mathbb{R}}$ has mean zero and variance one, then there exists a unique $v \in M_{\mathbb{R}}$ satisfying (2-5).

Given $\tau \in \mathscr{I}(\boxplus, \mathbb{R})$ and $\mu, v \in \mathcal{M}_{\mathbb{R}}$, we compare free Lévy process $v \boxplus \tau^{\boxplus t}$ and free convolution semigroup $\mu^{\boxplus(t+1)}$. If $\varphi_{\tau}\left(F_{v}(z)\right)=z-F_{\mu}(z)$, then $\tau \boxplus v=$ $\mu \boxminus \mu$, which implies that subordination function of $F_{\nu \boxplus\left(\tau^{\boxplus t}\right)}$ to $F_{v}$ is the same as the subordination function of $F_{\mu^{\boxplus(t+1)}}$ to $F_{\mu}$. The following theorem generalizes Theorem 2.7. The argument is similar to the proof of Theorem 1.6 in [Belinschi and Nica 2008b] (see also the proof of Lemma 3 in [Anshelevich 2012]); therefore we omit the proof.

Theorem 2.8. Given $\tau \in \mathscr{I}(\boxplus, \mathbb{R})$, and let $\mu$ and $v$ be a pair of probability measures on the real line such that

$$
\varphi_{\tau}\left(F_{v}(z)\right)=z-F_{\mu}(z), \quad z \in \mathbb{C}^{+} .
$$


Then we have

$$
\varphi_{\tau}\left(F_{\nu \boxplus(\tau \boxplus t)}(z)\right)=z-F_{\mathbb{B}_{t}(\mu)}(z), \quad t>0, z \in \mathbb{C}^{+} .
$$

Remark. Let $\tau=\gamma_{a, b}$ be the semicircular distribution with mean $a$ and variance $b$, and let $\mu, v$ be a pair of probability measures on the real line such that

$$
\varphi_{\tau}\left(F_{\nu}(z)\right)=z-F_{\mu}(z) .
$$

We first compute

$$
F_{\mu}(z)=z-\varphi_{\tau}\left(F_{\nu}(z)\right)=z-\left(a+\frac{b}{z}\right) \circ F_{\nu}(z)=z-a-b G_{v}(z) .
$$

Then, by Theorem 2.8,

$$
\begin{aligned}
F_{\mathbb{B}_{t}(\mu)}(z) & =z-\varphi_{\tau}\left(F_{\nu \boxplus \tau^{\boxplus t}}(z)\right) \\
& =z-\left(\left(a+\frac{b}{z}\right) \circ F_{\nu \boxplus \gamma_{a, b}^{\boxplus t}}\right)(z)=z-a-b G_{\nu \boxplus \gamma_{a, b}^{\boxplus t}}(z) .
\end{aligned}
$$

By (2-7) and the definition of Boolean convolution, we obtain

$$
F_{\left(\mathbb{B}_{t}(\mu)\right)^{\uplus t}}(z)=z-t a-t b G_{\nu \boxplus \gamma_{a, b}^{\boxplus t}}(z) .
$$

Equations (2-6), (2-7) and (2-8) were studied in [Anshelevich 2012]. As shown there (Proposition 1 and Example 1), we have $\left(\mathbb{B}_{t}(\mu)\right)^{\uplus t} \in \mathscr{I} \mathscr{D}(\boxplus, \mathbb{R})$, and $\left(\mathbb{B}_{t}(\mu)\right)^{\uplus t}=$ $(\tau \boxplus v)^{\boxplus t}=(\mu \boxplus \mu)^{\boxplus t}$. In fact, for all $\mu \in \mathcal{M}_{\mathbb{R}}$, we can deduce from (2-4) and the identity $\left(\mathbb{B}_{t}(\mu)\right)^{\uplus t}=\left(\mu^{\boxplus(1+t)}\right)^{\uplus t /(1+t)}$ that $\left(\mathbb{B}_{t}(\mu)\right)^{\uplus t}$ is the measure associated with the subordination function of $\mu^{\boxplus(1+t)}$ with respect to $\mu$ : $\left(\mathbb{B}_{t}(\mu)\right)^{\uplus t}=(\mu \boxminus \mu)^{\boxplus t}$.

\section{Multiplicative free convolution and multiplicative Boolean convolution on $\mu_{\mathbf{T}}$}

Given any two probability measures $\mu, v$ on $\mathrm{T}$, the unit circle of $\mathbb{C}$, we can define their multiplicative free convolution. We first recall the calculation of the multiplicative free convolution of two measures on $\mathrm{T}$ with nonzero means. Given $\mu \in M_{\mathrm{T}}$, we define

$$
\psi_{\mu}(z)=\int_{\mathrm{T}} \frac{t z}{1-t z} d \mu(t)
$$

and set $\eta_{\mu}(z)=\psi_{\mu}(z) /\left(1+\psi_{\mu}(z)\right)$. The following proposition characterizes the $\eta$-transforms of probability measures on $\mathrm{T}$.

Proposition 3.1 [Belinschi and Bercovici 2005]. If $\eta: \mathbb{D} \rightarrow \mathbb{C}$ is an analytic function, the following assertions are equivalent.

(1) There exists a probability measure $\mu \in M_{\mathrm{T}}$ such that $\eta=\eta_{\mu}$.

(2) $\eta(0)=0$, and $|\eta(z)|<1$ holds for all $z \in \mathbb{D}$. 
If $\mu \in \mathcal{M}_{\mathrm{T}} \cap M_{*}$, then $\eta_{\mu}^{\prime}(0)=\int_{\mathrm{T}} t d \mu(t) \neq 0$. Therefore, the inverse $\eta_{\mu}^{-1}$ is defined in a neighborhood of zero. We set $\Sigma_{\mu}(z)=\eta_{\mu}^{-1}(z) / z$. Given $\mu, v \in M_{\mathrm{T}} \cap \mu_{*}$, their multiplicative free convolution, which is denoted by $\mu \nabla v$, is the unique probability measure in $\mu_{\mathrm{T}} \cap \mu_{*}$ such that

$$
\Sigma_{\mu 凶 \nu}(z)=\Sigma_{\mu}(z) \Sigma_{\nu}(z)
$$

for $z$ in a neighborhood of zero.

It is known (see [Biane 1998; Belinschi and Bercovici 2007]) that there exist two analytic functions $\omega_{1}, \omega_{2}: \mathbb{D} \rightarrow \mathbb{D}$ such that

(1) $\omega_{1}(0)=\omega_{2}(0)=0$,

(2) $\eta_{\mu \bowtie v}(z)=\eta_{\mu}\left(\omega_{1}(z)\right)=\eta_{\nu}\left(\omega_{2}(z)\right)$.

A probability measure $\mu \in M_{\mathrm{T}}$ is said to be $\otimes$-infinitely divisible if for any positive integer $n$, there exists $\mu_{n} \in M_{\mathrm{T}}$ such that $\mu=\left(\mu_{n}\right)^{\bigotimes n}=\mu_{n} \otimes \cdots \otimes \mu_{n}$. It is shown in [Bercovici and Voiculescu 1992] that if $\mu \in \mathcal{M}_{T} \backslash \mathcal{M}_{*}$ is $\nabla$-infinitely divisible, then $\mu$ is the Haar measure on $\mathrm{T}$; and $\mu \in \mathcal{M}_{\mathrm{T}} \cap \mathcal{M}_{*}$ is $\otimes$-infinitely divisible if and only if there exists a function

$$
u(z)=\alpha i+\int_{\mathrm{T}} \frac{1+t z}{1-t z} d \sigma(t)
$$

such that $\Sigma_{\mu}(z)=\exp (u(z))$, where $\alpha \in \mathbb{R}$ and $\sigma$ is a finite positive measure on T. Equation (3-2) is the analogue of the Lévy-Khintchine formula for multiplicative free convolution on $\mathrm{T}$. The analogue of the normal distribution in this context is

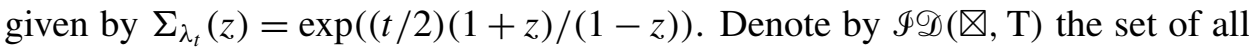
$\triangle$-infinitely divisible measures on $\mathrm{T}$.

Lemma 3.2. Let $\mu \in M_{\mathrm{T}} \cap M_{*}$ be $\nabla$-infinitely divisible.

(1) The function $H(z)=z \Sigma_{\mu}(z)$ is the left inverse of $\eta_{\mu}(z)$, that is $H\left(\eta_{\mu}(z)\right)=z$ for all $z \in \mathbb{D}$.

(2) The function $\eta_{\mu}$ extends to be a continuous function on $\overline{\mathbb{D}}$, and $\eta_{\mu}$ is one-to-one on $\overline{\mathbb{D}}$.

(3) The set $\left\{z \in \mathbb{D}:\left|z \Sigma_{\mu}(z)\right|<1\right\}$ is a simply connected domain which coincides with $\left\{\eta_{\mu}(z): z \in \mathbb{D}\right\}$, and its boundary is $\eta_{\mu}(T)$ which is a simple closed curve.

Proof. Observing that $H\left(\eta_{\mu}(z)\right)=z$ is valid in a neighborhood of zero, we obtain assertion (1) by analytic continuation.

Note that $H: \mathbb{D} \rightarrow \mathbb{C}$ satisfies the conditions in [Belinschi and Bercovici 2005, Proposition 4.5] and thus assertions (2) and (3) hold. 
Multiplicative free Brownian motion. For $\mu \in M_{\mathrm{T}}$ and $t>0$, we study the multiplicative free convolution $\mu \otimes \lambda_{t}$. We first concentrate on the case when $\mu$ has nonzero mean. The case when $\mu$ has mean zero will be studied in Subsection 3.2.

We start with the following result which is the multiplicative version of [Biane 1997b, Lemma 1].

Lemma 3.3. Given $\mu, v \in M_{\mathrm{T}} \cap \mu_{*}$, we have

$$
\eta_{\mu}(z)=\eta_{\mu 凶 v}\left(z \cdot \Sigma_{v}\left(\eta_{\mu}(z)\right)\right)
$$

for $z$ in a neighborhood of zero.

Proof. From (3-1), we find that

$$
\frac{\eta_{\mu 凶 \nu}^{-1}(z)}{z}=\frac{\eta_{\mu}^{-1}(z)}{z} \cdot \frac{\eta_{v}^{-1}(z)}{z}
$$

for $z$ in a neighborhood of zero, which we denote by $D_{0}$. We choose a subdomain $D_{1} \subset D_{0}$ such that $\eta_{\mu}\left(D_{1}\right) \subset D_{0}$. Replacing $z$ by $\eta_{\mu}(z)$ in (3-3), we obtain

$$
\frac{\eta_{\mu \bowtie \nu}^{-1}\left(\eta_{\mu}(z)\right)}{\eta_{\mu}(z)}=\frac{\eta_{\mu}^{-1}\left(\eta_{\mu}(z)\right)}{\eta_{\mu}(z)} \cdot \frac{\eta_{\nu}^{-1}\left(\eta_{\mu}(z)\right)}{\eta_{\mu}(z)}=\frac{z}{\eta_{\mu}(z)} \cdot \frac{\eta_{\nu}^{-1}\left(\eta_{\mu}(z)\right)}{\eta_{\mu}(z)}
$$

for $z \in D_{1}$. Note that $\eta_{v}^{-1}(z)=z \Sigma_{v}(z)$ for $z \in D_{0}$, and we then rewrite (3-4) as

$$
\eta_{\mu \bowtie v}^{-1}\left(\eta_{\mu}(z)\right)=z \Sigma_{v}\left(\eta_{\mu}(z)\right) \text {. }
$$

Applying $\eta_{\mu \bowtie v}$ on both sides of (3-5) yields

$$
\eta_{\mu}(z)=\eta_{\mu \bowtie v}\left(z \Sigma_{v}\left(\eta_{\mu}(z)\right)\right)
$$

for $z$ in a neighborhood of zero $D_{1}$.

For any $t>0$, we denote by $\eta_{t}: \mathbb{D} \rightarrow \mathbb{D}$ the subordination function of $\mu \otimes \lambda_{t}$ with respect to $\mu$. Since $\eta_{t}: \mathbb{D} \rightarrow \mathbb{D}$ is analytic and $\eta_{t}(0)=0$, Proposition 3.1 implies the existence of a probability measure $\rho_{t}$ such that $\eta_{\rho_{t}}(z)=\eta_{t}(z)$.

Lemma 3.4. The measure $\rho_{t}$ is $\bigotimes$-infinitely divisible and its $\Sigma$-transform is

$$
\Sigma_{\rho_{t}}(z)=\Sigma_{\lambda_{t}}\left(\eta_{\mu}(z)\right) .
$$

Proof. Define analytic function $\Phi_{t}: \mathbb{D} \rightarrow \mathbb{C}$ by $\Phi_{t}(z):=z \Sigma_{\lambda_{t}}\left(\eta_{\mu}(z)\right)$ for all $t>0$. By Lemma 3.3, we have

$$
\eta_{\mu}(z)=\eta_{\mu 凶 \lambda_{t}}\left(z \Sigma_{\lambda_{t}}\left(\eta_{\mu}(z)\right)\right)=\eta_{\mu 凶 \lambda_{t}}\left(\Phi_{t}(z)\right)
$$

which implies that

$$
\eta_{\mu 凶 \lambda_{t}}(z)=\eta_{\mu}\left(\eta_{t}(z)\right)=\eta_{\mu \bowtie \lambda_{t}}\left(\Phi_{t}\left(\eta_{t}(z)\right)\right) .
$$


Since $\eta_{\mu \otimes \lambda_{t}}$ is invertible in a neighborhood of zero, we have $\Phi_{t}\left(\eta_{t}(z)\right)=z$ in a neighborhood of zero.

We thus obtain $\eta_{\rho_{t}}^{-1}(z)=\eta_{t}^{-1}(z)=\Phi_{t}(z)$ holds for $z$ in a neighborhood of zero, which yields that

$$
\Sigma_{\rho_{t}}(z)=\frac{\eta_{\rho_{t}}^{-1}(z)}{z}=\Sigma_{\lambda_{t}}\left(\eta_{\mu}(z)\right)
$$

By the definition of the $\psi$ - and $\eta$-transforms, we have

$$
\Sigma_{\lambda_{t}}\left(\eta_{\mu}(z)\right)=\exp \left(\frac{t}{2} \int_{\mathrm{T}} \frac{1+\xi z}{1-\xi z} d \mu(\xi)\right) .
$$

The real part of the integrand in (3-7) is positive for all $z \in \mathbb{D}$; thus the assertion follows from (3-6) and [Bercovici and Voiculescu 1992, Theorem 6.7].

By (3-6), the right hand side of (3-7) is the Lévy-Khintchine representation of $\rho_{t}$. We can also write $\eta_{t}$ in terms of $\lambda_{t}$ and $\mu \otimes \lambda_{t}$. Replacing $z$ by $\eta_{\mu \otimes \lambda_{t}}(z)$ in the equation

we obtain

$$
\frac{\eta_{\mu \bowtie \lambda_{t}}^{-1}(z)}{z}=\frac{\eta_{\mu}^{-1}(z)}{z} \cdot \frac{\eta_{\lambda_{t}}^{-1}(z)}{z}
$$

$$
\frac{z}{\eta_{\mu \bowtie \lambda_{t}}(z)}=\frac{\eta_{t}(z)}{\eta_{\mu \bowtie \lambda_{t}}(z)} \cdot \Sigma_{\lambda_{t}}\left(\eta_{\mu \bowtie \lambda_{t}}(z)\right),
$$

which shows that

$$
\eta_{t}(z)=\frac{z}{\Sigma_{\lambda_{t}}\left(\eta_{\mu \otimes \lambda_{t}}(z)\right)} .
$$

Modified 9 -transform and subordination functions. Given $\mu \in M_{T} \backslash M_{*}$ and $v \in$ $\mu_{\mathrm{T}} \cap \mu_{*}$, it is known from [Biane 1998] that $\eta_{\mu 凶 v}$ is subordinated to $\eta_{\mu}$ and $\eta_{\nu}$. The subordination function for this case is generally not unique (see Example 3.5 below). However, we show that there is a nice subordination function, which we call the principal subordination function, uniquely determined by certain conditions. Using the principal subordination function, results related to subordination function in the case $\mu, v \in M_{\mathrm{T}} \cap \mathcal{M}_{*}$ can be extended to the case where $\mu \in \mathcal{M}_{\mathrm{T}} \backslash \mathcal{M}_{*}$ and $v \in M_{\mathrm{T}} \cap M_{*}$.

Let us first give an example which illustrates the non-uniqueness of subordination functions.

Example 3.5. For $k \in \mathbb{N}$, and let $\lambda^{(k)}=1 / k \sum_{n=0}^{k-1} \delta_{z_{n}}$, where $z_{n}=e^{2 \pi i n / k}$. We have $\psi_{\lambda^{(k)}}(z)=z^{k} /\left(1-z^{k}\right)$ and $\eta_{\lambda^{(k)}}(z)=z^{k}$. Given $v \in \mathcal{M}_{\mathrm{T}} \cap \mathcal{M}_{*}$, if $\omega: \mathbb{D} \rightarrow \mathbb{D}$ is a subordination function of $\eta_{\lambda^{(k)} \bowtie v}$ with respect to $\eta_{\lambda^{(k)}}$, then $\omega^{(n)}(z):=e^{2 \pi i n / k} \omega(z)$ is also a subordination function of $\eta_{\lambda^{(k)} \bowtie v}$ with respect to $\eta_{\lambda^{(k)}}$ for all integer $0<n<k$. 
We now introduce the modified $\mathscr{Y}$-transform. Given two free random variables $x$ and $y$ in a $\mathrm{W}^{*}$-probability space $(\mathscr{A}, \phi)$, such that $\phi(x)=0$ and $\phi(y) \neq 0$, we can not directly apply Voiculescu's $\mathscr{S}$-transform ( $\Sigma$-transform) to calculate the distribution of $x y$. N. Raj Rao and R. Speicher [2007] introduce a new transform, which we call the modified $\mathscr{S}$-transform, to deal with this case. They apply the modified $\mathscr{Y}$-transform to study the distribution of $x y$ where $x, y$ are free self-adjoint random variables such that $\phi(x)=0, \phi(y) \neq 0$. For nonzero self-adjoint operator $x$, we have $\phi\left(x^{2}\right) \neq 0$. Assume that $\phi(x)=\cdots=\phi\left(x^{k-1}\right)=0$ and $\phi\left(x^{k}\right) \neq 0$. Arizmendi [2012] observe that we can calculate the distribution of $x y$ using the idea in [Rao and Speicher 2007]. We present the details of their work for reader's convenience.

We first recall some definitions. For $\mu \in M_{\mathrm{T}} \cap \mu_{*}$, we have $\psi_{\mu}(0)=0$ and $\psi_{\mu}^{\prime}(0) \neq 0$. It follows that there exists a function $\chi_{\mu}(z)$, which is analytic in a neighborhood of zero, such that

$$
\psi_{\mu}\left(\chi_{\mu}(z)\right)=\chi_{\mu}\left(\psi_{\mu}(z)\right)=z
$$

for sufficiently small $z$. The usual $\mathscr{S}$-transform is defined by

We then have

$$
\mathscr{S}_{\mu}(z)=\frac{z+1}{z} \chi_{\mu}(z)
$$

$$
\Sigma_{\mu}(z)=\mathscr{Y}_{\mu}\left(\frac{z}{1-z}\right), \quad \eta_{\mu}^{-1}(z)=\chi\left(\frac{z}{1-z}\right)
$$

We set

$$
M_{\mathrm{T}}^{k}=\left\{\mu \in \mathcal{M}_{\mathrm{T}}: \int_{\mathrm{T}} t^{n} d \mu(t)=0 \text { for } 1 \leq n<k, \text { and } \int_{\mathrm{T}} t^{k} d \mu(t) \neq 0\right\} .
$$

Then, for $\mu \in M_{\mathrm{T}}^{k}$, we have

$$
\left\{\begin{array}{l}
\psi_{\mu}^{\prime}(0)=\cdots=\psi_{\mu}^{(k-1)}(0)=0=\eta_{\mu}^{\prime}(0)=\cdots=\eta_{\mu}^{(k-1)}(0), \\
\psi_{\mu}^{(k)}(0) \neq 0 \text { and } \eta_{\mu}^{(k)} \neq 0 .
\end{array}\right.
$$

For $\mu \in \mathcal{M}_{\mathrm{T}}^{k}, v \in \mathcal{M}_{\mathrm{T}} \cap \mathcal{M}_{*}$, from the definition of free independence, we deduce that $\mu \otimes v \in \mu_{\mathrm{T}}^{k}$.

We recall the following classical result in complex analysis; see, for example, [Hille 1959].

Theorem 3.6. If $f(z)$ is holomorphic in $|z|<R$, and suppose that

$$
f(0)=f^{\prime}(0)=\cdots=f^{(k-1)}(0)=0, \quad f^{(k)} \neq 0 .
$$

Then for small values of $w \neq 0$ the equation

$$
f(z)=w
$$


has $k$ roots $z_{1}(w), \ldots, z_{k}(w)$, which tend to zero when $w$ tends to zero. Moreover, there exists a function $g(w)$, holomorphic for $w$ sufficiently small with $g(0)=0$ and $g^{\prime}(0) \neq 0$, such that for any fixed small values $w \neq 0$,

$$
z_{j}(w)=g\left(\omega^{j} w^{1 / k}\right), \quad \omega=e^{2 \pi i / k}, \quad 0 \leq \arg \omega^{1 / k}<\frac{2 \pi}{k},
$$

if we put those roots in a certain order.

Remark. The converse of Theorem 3.6 is also true. More precisely, given a function $g(w)$ that is holomorphic for small enough $w$ and satisfies $g(0)=0$ and $g^{\prime}(0) \neq 0$, if we set

$$
z_{j}(w)=g\left(\omega^{j} w^{1 / k}\right), \quad \omega=e^{2 \pi i / k}, \quad 0 \leq \arg \omega^{1 / k}<\frac{2 \pi}{k}
$$

for $j=1, \ldots, k$, then $z_{1}(w), \ldots, z_{k}(w)$ are the roots of the equation

$$
F^{k}(z)=w
$$

where $F$ is a holomorphic function defined in a neighborhood of the zero such that $F(g(w))=w$.

For $j=1, \ldots, k$, denote $D_{j, r}=\left\{\omega^{j} z: 0 \leq \arg (z)<2 \pi / k,|z|<r\right\}$. We record the following result for convenience.

Proposition 3.7. Under the assumption of Theorem 3.6, we have $z_{j}(f(z))=z$ for $z \in g\left(D_{j, r}\right)$ for $r$ sufficiently small.

Given $\mu \in M_{\mathrm{T}}^{k}$, and by Theorem 3.6, we know that there exist $k$ functions represented by the power series in $z^{1 / k}$ such that

$$
\psi_{\mu}\left(\chi_{\mu}^{(j)}(z)\right)=z
$$

for $z$ sufficiently small. Moreover, there exists a function $g_{\mu}(w)$ holomorphic in a neighborhood of the zero, such that for $j=1, \ldots, k$,

$$
\chi_{\mu}^{(j)}(z)=g_{\mu}\left(\omega^{j} z^{1 / k}\right),
$$

where $\omega=e^{2 \pi i / k}, 0 \leq \arg z^{1 / k}<2 \pi / k$.

Definition 3.8. Given $\mu \in M_{\mathrm{T}}^{k}$. Let $\chi_{\mu}^{(j)}$ be the inverse function of $\psi_{\mu}$ in (3-10),

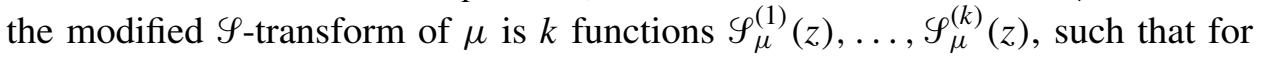
$j=1, \ldots, k$,

$$
\varphi_{\mu}^{(j)}(z)=\chi_{\mu}^{(j)}(z) \cdot \frac{1+z}{z} .
$$

Given $\mu \in M_{\mathrm{T}}^{k}$ and $v \in \mathcal{M}_{\mathrm{T}} \cap M_{*}$, we set

$$
\mathscr{S}^{(j)}(z)=\mathscr{Y}_{\mu}^{(j)}(z) \cdot \mathscr{S}_{\nu}(z)
$$


and compute

$$
\begin{aligned}
\chi^{(j)}(z) & =\mathscr{Y}^{(j)}(z) \cdot \frac{z}{1+z}=\mathscr{Y}_{\mu}^{(j)}(z) \cdot \mathscr{Y}_{\nu}(z) \cdot \frac{z}{1+z} \\
& =\chi_{\mu}^{(j)}(z) \cdot \mathscr{S}_{\nu}(z)=g_{\mu}\left(\omega^{j} z^{1 / k}\right) \cdot \mathscr{S}_{\nu}(z)=g\left(\omega^{j} z^{1 / k}\right),
\end{aligned}
$$

where $g(z)=g_{\mu}(z) \cdot \mathscr{Y}_{v}\left(z^{k}\right)$ is a function such that $g(0)=0, g^{\prime}(0) \neq 0$. From the remark after Theorem 3.6, we deduce that for different $j$, there exists the same left inverse $\psi$ such that $\psi\left(\chi^{(j)}(z)\right)=z$. Therefore, we have the following proposition.

Proposition 3.9. Given $\mu \in M_{\mathrm{T}}^{k}$ and $v \in \mathcal{M}_{\mathrm{T}} \cap \mathcal{M}_{*}$, for $1 \leq j \leq k$, let

$$
\mathscr{S}^{(j)}(z)=\mathscr{\varphi}_{\mu}^{(j)}(z) \cdot \mathscr{Y}_{\nu}(z), \quad \chi^{(j)}(z)=\mathscr{S}^{(j)}(z) \cdot \frac{z}{1+z} .
$$

Then there exists a unique holomorphic function $\psi$ defined in a neighborhood of the zero such that

$$
\psi\left(\left(\chi^{(j)}\right)(z)\right)=z
$$

Theorem 3.10 [Rao and Speicher 2007; Arizmendi 2012]. Given $\mu \in \mu_{\mathrm{T}}^{k}$ and $v \in M_{\mathrm{T}} \cap M_{*}$, we have

$$
\mathscr{S}_{\mu \bowtie v}^{(j)}(z)=\mathscr{Y}_{\mu}^{(j)}(z) \cdot \mathscr{Y}_{\nu}(z), \quad j=1, \ldots, k,
$$

where the modified $\mathscr{Y}$-transforms are listed in a certain order.

Because of Proposition 3.9 and Theorem 3.10, for fixed $\mu \in \mathcal{M}_{\mathrm{T}}^{k}$ and $v \in \mathcal{M}_{\mathrm{T}} \cap \mathcal{M}_{*}$, we denote

$$
\psi(z)=\psi_{\mu \bowtie v}(z) \quad \text { and } \quad \chi^{(j)}(z)=\chi_{\mu \bowtie v}^{(j)}(z),
$$

and we also denote $g(z)=g_{\mu}(z) \cdot \mathscr{Y}_{\nu}\left(z^{k}\right)$ as in (3-11).

Given $\mu \in M_{\mathrm{T}}^{k}, v \in M_{\mathrm{T}} \cap \mathcal{M}_{*}$, we set $\iota_{\mu}^{(j)}(z)=\chi_{\mu}^{(j)}(z /(1-z))$ and $\iota_{v}(z)=$ $\chi_{v}(z /(1-z))$. Theorem 3.10 implies that

$$
\chi_{\mu \bowtie v}^{(j)}(z)=\chi_{\mu}^{(j)}(z) \cdot \chi_{v}(z) \cdot \frac{1+z}{z} .
$$

We also have $\chi_{\mu}^{(j)}(z)=g_{\mu}\left(\omega^{j} z^{1 / k}\right)$ and $\chi_{\mu \unrhd v}^{(j)}(z)=g_{\mu}\left(\omega^{j} z^{1 / k}\right) \cdot \mathscr{Y}_{v}(z)=g\left(\omega^{j} z^{1 / k}\right)$. Substituting $z$ by $\psi_{\mu \otimes v}(z)$ in (3-14), and applying Proposition 3.7, we find that

$$
z=\chi_{\mu \bowtie v}^{(j)}\left(\psi_{\mu \bowtie v}(z)\right)=\chi_{\mu}^{(j)}\left(\psi_{\mu \bowtie v}(z)\right) \cdot \chi_{v}\left(\psi_{\mu \bowtie v}(z)\right) \cdot \frac{1+\psi_{\mu \bowtie v}(z)}{\psi_{\mu \bowtie v}(z)},
$$

where $z \in g\left(D_{j, r}\right)$ for $r$ sufficiently small. Thus

$$
z \eta_{\mu 凶 v}=\iota_{\mu}^{(j)}\left(\eta_{\mu \bowtie v}\right) \cdot \iota_{v}\left(\eta_{\mu 凶 v}\right)
$$

holds in the same domain. 
We can now utilize the argument in [Belinschi and Bercovici 2007] to prove the existence of subordination function of $\eta_{\mu \bowtie v}$ with respect to $\eta_{\mu}$ for $\mu \in M_{\mathrm{T}}^{k}, v \in$ $\mathcal{M}_{\mathrm{T}} \cap \mathcal{M}_{*}$. Note that part of the following result is known in [Biane 1998].

Theorem 3.11. Given $\mu \in \mathcal{M}_{\mathrm{T}}^{k}, v \in \mathcal{M}_{\mathrm{T}} \cap \mathcal{M}_{*}$, there exists two unique analytic functions $\omega_{1}, \omega_{2}: \mathbb{D} \rightarrow \mathbb{D}$ such that

(1) $\omega_{1}(0)=\omega_{2}(0)=0$;

(2) $\eta_{\mu \bowtie v}(z)=\eta_{\mu}\left(\omega_{1}(z)\right)=\eta_{v}\left(\omega_{2}(z)\right)$,

(3) $\omega_{1}(z) \omega_{2}(z)=z \eta_{\mu 凶 v}(z)$ for all $z \in \mathbb{D}$.

Proof. Since $\eta_{\mu}(0)=0, \eta_{\nu}(0)=0$, we can write $\eta_{\mu}(z)=z f_{1}(z), \eta_{\nu}(z)=z f_{2}(z)$ for two analytic functions $f_{1}, f_{2}: \mathbb{D} \rightarrow \mathbb{D}$. Fix $1 \leq j \leq k$, set $\omega_{1}(z)=\iota_{\mu}^{(j)}\left(\eta_{\mu \Downarrow v}(z)\right)$, $\omega_{2}(z)=\iota_{v}\left(\eta_{\mu \bowtie v}(z)\right)$ defined in $g\left(D_{j, r}\right)$ for $r$ sufficiently small.

By (3-15), we have

$$
z \eta_{\mu \bowtie v}(z)=\omega_{1}(z) \omega_{2}(z)
$$

for $z \in g\left(D_{j, r}\right)$. We thus obtain

$$
\omega_{1}(z)=\frac{z \eta_{\mu \bowtie v}}{\omega_{2}(z)}=\frac{z \eta_{v}\left(\omega_{2}(z)\right)}{\omega_{2}(z)}=z f_{2}\left(\omega_{2}(z)\right) .
$$

Similarly, we have $\omega_{2}(z)=z f_{1}\left(\omega_{1}(z)\right)$ for $z \in g\left(D_{j, r}\right)$. Regarding $\omega_{1}(z), \omega_{2}(z)$ as Denjoy-Wolff points, the same argument as in [Belinschi and Bercovici 2007] implies $\omega_{1}, \omega_{2}$ can be extended analytically to $\mathbb{D}$. By the uniqueness of DenjoyWolff points, $\omega_{1}, \omega_{2}$ does not depend on the choice of $j$.

By the definitions of $\iota_{\mu}^{(j)}, \iota_{\nu}$, we have $\eta_{\mu}\left(\omega_{1}(z)\right)=\eta_{\nu}\left(\omega_{2}(z)\right)=\eta_{\mu \bowtie v}$ for $z$ in $g\left(D_{j, r}\right)$. Thus (2) and (3) hold by analytic continuation. Since $\eta_{v}^{\prime}(0) \neq 0, \eta_{v}$ is locally invertible near the origin and therefore $\omega_{2}$ is unique. Finally (3) implies the uniqueness of $\omega_{1}$.

Since $\mu \in M_{\mathrm{T}}^{k}$ and $\mu \otimes v \in M_{\mathrm{T}}^{k}$, we have $\omega_{1}^{\prime}(0) \neq 0$, where $\omega_{1}$ is given in Theorem 3.11.

Definition 3.12. For $\mu \in \mathcal{M}_{\mathrm{T}}^{k}, v \in \mathcal{M}_{\mathrm{T}} \cap \mathcal{M}_{*}$, the subordination $\omega_{1}$ satisfying the relations (1), (2) and (3) in Theorem 3.11 is called the principal subordination function of $\eta_{\mu \bowtie v}$ with respect to $\eta_{\mu}$. The measure $\rho \in M_{\mathrm{T}} \cap \mu_{*}$ satisfying $\eta_{\rho}(z)=$ $\omega_{1}(z)$ is called the principal subordination distribution of $\eta_{\mu \bowtie \nu}$ with respect to $\eta_{\mu}$.

Note that for $\mu, v \in M_{\mathrm{T}} \cap M_{*}$, the principal subordination function of $\eta_{\mu \bowtie v}$ with respect to $\eta_{\mu}$ is the usual subordination function.

The following result might be obtained by approximation. We provide a direct proof. 
Corollary 3.13. Given $\mu \in M_{\mathrm{T}}^{k}, v \in M_{\mathrm{T}} \cap \mu_{*}$, let $\rho$ be the principal subordination distribution of $\eta_{\mu \bowtie v}$ with respect to $\eta_{\mu}$, we have

$$
\Sigma_{\rho}(z)=\Sigma_{\nu}\left(\eta_{\mu}(z)\right)
$$

In particular, if $v \in \mathscr{\mathscr { D }}(\bigotimes, \mathrm{T})$, we have $\rho \in \mathscr{\mathscr { D }}(\bigotimes, \mathrm{T})$.

Proof. By choosing a sequence $\mu_{n} \in \mathcal{M}_{\mathrm{T}} \cap \mathcal{M}_{*}$ such that $\mu_{n}$ converges to $\mu$ weakly, Lemma 3.3 implies

$$
\eta_{\mu}(z)=\eta_{\mu \bowtie v}\left(z \Sigma_{v}\left(\eta_{\mu}(z)\right)\right)
$$

for $z$ in a neighborhood of zero.

Set $\Phi(z)=z \Sigma_{v}\left(\eta_{\mu}(z)\right)=z \cdot \mathscr{Y}_{\nu}\left(\psi_{\mu}(z)\right)$, and we thus have

$$
\eta_{\mu 凶 \nu}(z)=\eta_{\mu}\left(\omega_{1}(z)\right)=\eta_{\mu \bowtie v}\left(\Phi\left(\omega_{1}(z)\right)\right)=\eta_{\mu \bowtie v}\left(\Phi\left(\eta_{\rho}(z)\right)\right) .
$$

Fix $1 \leq j \leq k$, we claim that if $z \in g\left(D_{j, r}\right)$, then $\Phi\left(\omega_{1}(z)\right)=z$. Indeed, for $0 \leq \arg \left(w^{1 / k}\right)<2 \pi / k$ and $z=g\left(\omega^{j} w^{1 / k}\right)=\chi^{(j)}(w)$, using the construction of $\omega_{1}$ in Theorem 3.11, we have

$$
\omega_{1}(z)=\iota_{\mu}^{(j)}\left(\eta_{\mu \Downarrow v}(z)\right)=\chi_{\mu}^{(j)}\left(\psi_{\mu \Downarrow v}(z)\right) .
$$

From (3-11) and (3-13), we have

$$
\left.\chi^{(j)}(w)=g\left(\omega^{j} w^{1 / k}\right)\right) \quad \text { and } \quad \psi_{\mu \bowtie v}\left(\chi^{(j)}(w)\right)=w .
$$

Equations (3-17) and (3-18) imply that $\left.\omega_{1}\left(g\left(\omega^{j} w^{1 / k}\right)\right)\right)=\chi_{\mu}^{(j)}(w)$.

Note that $\psi_{\mu}\left(\omega_{1}\left(g\left(\omega^{j} w^{1 / k}\right)\right)\right)=\psi_{\mu \bowtie v}\left(g\left(\omega^{j} w^{1 / k}\right)\right)=w$. Thus we obtain

$$
\Phi\left(\omega_{1}(z)\right)=\Phi\left(\omega_{1}\left(g\left(\omega^{j} w^{1 / k}\right)\right)\right)=\chi_{\mu}^{(j)}(w) \mathscr{S}_{v}(w)=\chi^{(j)}(w)=z .
$$

The above claim, (3-16) and Proposition 3.7 imply

$$
z=\Phi\left(\omega_{1}(z)\right)=\Phi\left(\eta_{\rho}(z)\right)
$$

for $z \in g\left(D_{j, r}\right)$. We conclude that $\Sigma_{\rho}(z)=\Phi(z) / z=\Sigma_{\nu}\left(\eta_{\mu}(z)\right)$ for $z$ in a small neighborhood of zero by applying the above argument for all $1 \leq j \leq k$.

If $v \in \mathscr{D}(\otimes, T)$, then by [Bercovici and Voiculescu 1993, Theorem 6.7], there exists an analytic function $u(z)$ defined in $\mathbb{D}$ such that $\Sigma_{\nu}(z)=\exp (u(z))$ and $\Re u(z) \geq 0$ for all $z \in \mathbb{D}$. Thus $\Sigma_{v}\left(\eta_{\mu}(z)\right)=\exp \left(u\left(\eta_{\mu}(z)\right)\right)$ and $\Re\left(u\left(\eta_{\mu}(z)\right)\right) \geq 0$ for all $z \in \mathbb{D}$, and then the second assertion follows from [Bercovici and Voiculescu 1993, Theorem 6.7].

Remark. If $k=1$, noticing that $\mathcal{M}_{\mathrm{T}}^{k}=\mathcal{M}_{\mathrm{T}} \cap \mathcal{M}_{*}$, the modified $\mathscr{S}$-transform is the usual $\mathscr{Y}$-transform. We see that Corollary 3.14 holds when $\mu, v \in M_{\mathrm{T}} \cap \mathcal{M}_{*}$.

The following result is the multiplicative analogue of Lemma 2.6. 
Proposition 3.14. Given $\rho, \tau \in \mathcal{M}_{\mathrm{T}} \cap \mu_{*}$, let $\sigma$ be a measure in $\mathcal{M}_{\mathrm{T}} \cap \mathcal{M}_{*}$ such that $\eta_{\rho \bigotimes \tau}(z)=\eta_{\rho}\left(\eta_{\sigma}(z)\right)$. If $\sigma \in \mathscr{I D}(\bigotimes, \mathrm{T})$, then $\rho \otimes \tau^{\otimes t}$ can be defined for all $t \geq 0$ in the sense that $\Sigma_{\rho \bigotimes(\tau \otimes t)}(z)=\Sigma_{\rho}(z)\left(\Sigma_{\tau}(z)\right)^{t}$.

Proof. For $t>0$, there exists $\mu_{t} \in \mathcal{M}_{\mathrm{T}} \cap \mathcal{M}_{*}$ such that

$$
\eta_{\mu_{t}}(z)=\eta_{\rho}\left(\eta_{\sigma^{\otimes t}}\right) .
$$

Using a similar argument as in the proof of Lemma 2.6 and applying Corollary 3.13, we can find that $\Sigma_{\mu_{t}}(z)=\Sigma_{\rho}(z)\left(\Sigma_{\tau}(z)\right)^{t}$.

Semigroups related to multiplicative free convolution. Recall that

$$
\mathcal{M}_{\mathrm{T}}^{*}=\left\{\mu \in \mathcal{M}_{\mathrm{T}} \cap \mathcal{M}_{*}: \eta_{\mu}(z) \neq 0 \text { for all } z \in \mathbb{D} \backslash\{0\}\right\} .
$$

Let $\mu \in \mathcal{M}_{\mathrm{T}}^{*}$ and $t>1$ be given, and let $u$ be an analytic function such that $z /\left(\eta_{\mu}(z)\right)=$ $e^{u(z)}$ for $z$ in a neighborhood of zero. Set $H_{t}(z)=z e^{(t-1) u(z)}=z\left[z /\left(\eta_{\mu}(z)\right)\right]^{t-1}$. It is shown in [Belinschi and Bercovici 2005] that $H_{t}$ has a right inverse $\omega_{t}: \mathbb{D} \rightarrow \mathbb{D}$ such that $H_{t}(\omega(z))=z$, and there exists a probability measure $\mu^{\bigotimes t} \in \mathcal{M}_{\mathrm{T}}^{*}$ such that

(1) $\eta_{\mu} \otimes_{t}(z)=\eta_{\mu}\left(\omega_{t}(z)\right)$ and $\Sigma_{\mu t}(z)=\left(\Sigma_{\mu}(z)\right)^{t}$,

(2) $\omega_{t}(z)=\eta_{\mu^{\otimes t}}(z)\left[z / \eta_{\mu} \otimes_{t}(z)\right]^{1 / t}$ for $z \in \mathbb{D}$, where the power is chosen so that the equation holds.

Observe that for each $t>0$, by Proposition 3.1, there exists a probability measure $\sigma_{t} \in M_{\mathrm{T}}$ such that $\eta_{\sigma_{t}}(z)=\omega_{t+1}(z)$. It turns out that $\sigma_{t}$ is $\nabla$-infinitely divisible and its $\Sigma$-transform is $\Sigma_{\sigma_{t}}(z)=\left[z / \eta_{\mu}(z)\right]^{t}$, which can be obtained by applying the same argument as in the proof of Lemma 3.4.

The following result is a partial converse of [Belinschi and Bercovici 2005, Theorem 3.5].

Theorem 3.15. Given $\mu \in M_{\mathrm{T}} \cap M_{*}$, assume that for any $t>1$, there exists a probability measure $\mu_{t} \in M_{\mathrm{T}}$ such that

$$
\Sigma_{\mu_{t}}(z)=\left(\Sigma_{\mu}(z)\right)^{t} .
$$

Assume in addition that $\mu_{t}$ is subordinated with respect to $\mu$ for all $t>1$. Then $\eta_{\mu}(z) \neq 0$ for all $z \in \mathbb{D} \backslash\{0\}$, that is $\mu \in M_{\mathrm{T}}^{*}$.

Proof. For each $t>1$, we denote by $\omega_{t}$ the subordination function of $\mu_{t}$ to $\mu$. Observing that $\mu_{t} \in \mathcal{M}_{*}$ and $\omega_{t}^{\prime}(0) \neq 0$, for each $t>1$, there exists a probability measure $\sigma_{t-1} \in M_{\mathrm{T}} \cap M_{*}$ such that $\eta_{\sigma_{t-1}}(z)=\omega_{t}(z)$. We rewrite (3-19) as

$$
\frac{\eta_{\mu_{t}}^{-1}(z)}{z}=\left[\frac{\eta_{\mu}^{-1}(z)}{z}\right]^{t}
$$

for $z$ in a neighborhood of zero. 
Note that $\omega_{t}^{-1}(z)=\eta_{\mu_{t}}^{-1}\left(\eta_{\mu}(z)\right)$ for $z$ in a neighborhood of zero. Replacing $z$ by $\eta_{\mu}(z)$ in (3-20), we obtain

$$
\frac{\omega_{t}^{-1}(z)}{\eta_{\mu}(z)}=\frac{\eta_{\mu_{t}}^{-1}\left(\eta_{\mu}(z)\right)}{\eta_{\mu}(z)}=\left[\frac{\eta_{\mu}^{-1}\left(\eta_{\mu}(z)\right)}{\eta_{\mu}(z)}\right]^{t}=\left[\frac{z}{\eta_{\mu}(z)}\right]^{t},
$$

which implies

$$
\frac{\omega_{t}^{-1}(z)}{z}=\left[\frac{z}{\eta_{\mu}(z)}\right]^{t-1} .
$$

Given $t>0$, we thus have $\Sigma_{\sigma_{t}}(z)=\left[z / \eta_{\mu}(z)\right]^{t}$ for $z$ in a neighborhood of zero. Therefore $\sigma_{t}$ is $\bigotimes$-infinitely divisible.

By [Bercovici and Voiculescu 1992, Theorem 6.7], there exists an analytic function $u(z)$ in $\mathbb{D}$ such that $\Re u(z) \geq 0$ if $z \in \mathbb{D}$ and $\Sigma_{\sigma_{1}}(z)=\exp (u(z))$. We thus obtain $z / \eta_{\mu}(z)=\exp (u(z))$, which implies that $\eta_{\mu}(z) \neq 0$ for all $z \in \mathbb{D}$.

It was pointed out in [Belinschi and Bercovici 2005] that $\mu^{\bigotimes t}$ is only determined up to a rotation by a multiple of $2 \pi t$. Note that $\omega_{t}$ and $\sigma_{t}$ are determined by the choice of $\mu^{\bigotimes t}$.

Multiplicative Boolean convolution and the Bercovici-Pata bijection. Multiplicative Boolean convolution on T was studied by Franz [2008]. Let $\mu \in \mathcal{M}_{\mathrm{T}}$, and we set $k_{\mu}(z)=z / \eta_{\mu}(z)$. Given two probability measures $\mu, v \in \mathcal{M}_{\mathrm{T}}$, their multiplicative Boolean convolution $\mu \otimes v$ is a probability measure on $\mathrm{T}$ such that

$$
k_{\mu 凶 v}(z)=k_{\mu}(z) k_{v}(z)
$$

for all $z \in \mathbb{D}$.

A probability $\mu \in M_{\mathrm{T}}$ is said to be $\otimes$-infinitely divisible, if for any positive integer $n$, there exists $\mu_{n} \in \mathcal{M}_{\mathrm{T}}$ such that $\mu=\left(\mu_{n}\right)^{\bigotimes n}$. Let $P_{0}$ be the Haar measure. It is shown in [Franz 2008] that $\mu \in \mathcal{M}_{\mathrm{T}} \backslash\left\{P_{0}\right\}$ is $凶$-infinitely divisible if and only if $\eta_{\mu}^{\prime}(0) \neq 0$ and $\eta_{\mu} \neq 0$ for all $z \in \mathbb{D} \backslash\{0\}$, that is $\mu \in \mathcal{M}_{\mathrm{T}}^{*}$, which is equivalent to

$$
k_{\mu}(z)=\exp \left(b i+\int_{\mathrm{T}} \frac{1+\xi z}{1-\xi z} d \tau_{\mu}(\xi)\right),
$$

where $b \in \mathbb{R}$ and $\tau_{\mu}$ is a finite measure on T. Equation (3-21) is the analogue of the Lévy-Khintchine formula in this context.

The multiplicative Bercovici-Pata bijection from $\otimes$ to $\otimes$ was studied in [Wang 2008]. Denote the set of all $\bowtie$-infinitely divisible measures on $\mathrm{T}$ by $\mathscr{I} \mathscr{D}(\otimes, T)$, and the multiplicative Bercovici-Pata bijection from $\otimes$ to $\otimes$ by $\mathbb{M}$. Then we have $k_{\mu}(z)=\Sigma_{\mathbb{M}(\mu)}(z)$.

Given $\mu \in \mathscr{I} \mathscr{D}(\otimes, \mathrm{T}) \backslash P_{0}=\mathcal{M}_{\mathrm{T}}^{*}$, let $\omega_{2}$ be the subordination function of $\mu^{\bigotimes 2}$ with respect to $\mu$, and let $\sigma$ be the probability measure on T such that $\eta_{\sigma}(z)=\omega_{2}(z)$. 
Then $\sigma$ is $\bigotimes$-infinitely divisible and its $\Sigma$-transform is $\Sigma_{\sigma}(z)=z / \eta_{\mu}(z)=k_{\mu}(z)$. Therefore, $\sigma$ is the same as $\mathbb{M}(\mu)$. Since $P_{0} \otimes P_{0}=P_{0}$ and $\eta_{P_{0}}=z$, the subordination function of $P_{0} \otimes P_{0}$ with respect to $P_{0}$ is the identity map $z$, and the measure associated with the identity map $z$ is $P_{0}$. To summarize:

Corollary 3.16. Given $\mu \in \mathscr{I} \mathscr{D}(\bowtie, T)$, let $\omega_{2}$ be the subordination function of $\mu^{\otimes 2}$ with respect to $\mu$, and let $\sigma$ be the probability measure on $\mathrm{T}$ such that $\eta_{\sigma}(z)=\omega_{2}(z)$. Then $\sigma=\mathbb{M}(\mu)$, where $\mathbb{M}$ is the multiplicative Bercovici-Pata bijection from $\otimes$ to $\otimes$.

Proposition 3.17. If $\mu \in \mathcal{M}_{\mathrm{T}}$, the following are equivalent.

(1) $\mu \in \mathscr{I} \mathscr{D}(\mathrm{T}, \otimes)$.

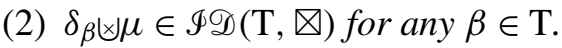

Proof. It is enough to prove that (1) implies (2) for $\mu \in \mathcal{M}_{\mathrm{T}} \cap M_{*}$. Observing that $\eta_{\delta_{\beta} \circlearrowright \mu}(z)=\beta \cdot \eta_{\mu}(z)$, we thus have

$$
\Sigma_{\delta_{\beta} 凶 \mu}(z)=\frac{\eta_{\delta_{\beta} 凶 \mu}^{-1}(z)}{z}=\frac{\eta_{\mu}^{-1}(\bar{\beta} z)}{z}=\bar{\beta} \cdot \Sigma_{\mu}(\bar{\beta} z) .
$$

The result follows from the Lévy-Khintchine formula for the multiplicative free convolution on $\mathrm{T}$.

An analogue of equations studied by Belinschi and Nica. In this subsection, we prove our Theorem 1.1. Recall that $\lambda_{t}$ is the free multiplicative analogue of the normal distribution on $\mathrm{T}$, the unit circle of $\mathbb{C}$, with $\Sigma_{\lambda_{t}}(z)=\exp ((t / 2)(1+z) /(1-z))$ and we set $\lambda=\lambda_{1}$. For $\mu \in M_{\mathrm{T}}$, we denote $m_{1}(\mu)=\int_{\mathrm{T}} \xi d \mu(\xi)$.

Proposition 3.18. Given $\mu \in M_{\mathrm{T}}^{*}$ and a finite measure $\tau$ on $\mathrm{T}$, define an analytic map $u$ by

$$
u(z)=b i+\int_{\mathrm{T}} \frac{1+\xi z}{1-\xi z} d \tau(\xi), \quad z \in \mathbb{D},
$$

where $b \in[0,2 \pi)$. If $k_{\mu}(z)=z / \eta_{\mu}(z)=\exp u(z)$, then $b=\arg 1 / m_{1}(\mu) \in[0,2 \pi)$ and $\tau(\mathrm{T})=\ln \left|1 / m_{1}(\mu)\right|$. In particular, there exists a probability measure $v \in M_{\mathrm{T}}$ such that $k_{\mu}(z)=\Sigma_{\lambda}\left(\eta_{v}(z)\right)$ if and only if $m_{1}(\mu)=e^{-1 / 2}$.

Proof. By definition, we have

$$
k_{\mu}(0)=\lim _{z \rightarrow 0} \frac{z}{\eta_{\mu}(z)}=\frac{1}{\eta_{\mu}^{\prime}(0)}=\frac{1}{m_{1}(\mu)} .
$$

Since $u(0)=b i+\tau(\mathrm{T})$, we obtain

$$
b=\arg \left(\frac{1}{m_{1}(\mu)}\right) \quad \text { and } \quad \tau(\mathrm{T})=\ln \left|\frac{1}{m_{1}(\mu)}\right| .
$$


The first assertion follows.

By (3-7), we have

$$
\Sigma_{\lambda}\left(\eta_{v}(z)\right)=\exp \left(\frac{1}{2} \int_{\mathrm{T}} \frac{1+\xi z}{1-\xi z} d \nu(\xi)\right) .
$$

Noticing that $k_{\mu}$ has the Herglotz representation as (3-21), we conclude that $k_{\mu}(z)$ can be written in the form of $\Sigma_{\lambda}\left(\eta_{\nu}(z)\right)$ for a probability measure $v$ on $\mathrm{T}$ if and only if $\ln \left(1 / m_{1}(\mu)\right)=1 / 2$. This implies the second half of the assertion.

For $\mu \in \mathscr{I} \mathscr{D}(\otimes, \mathrm{T}) \backslash P_{0}=M_{\mathrm{T}}^{*}$ with $m_{1}(\mu)>0$, let $u(z)$ be the analytic function satisfying $k_{\mu}(z)=\exp (u(z))$ and $u(0)>0$. Given $t>1$, let $H_{t}(z)=z \exp ((t-$ $1) u(z)$ ), and denote its right inverse by $\omega_{t}: \mathbb{D} \rightarrow \mathbb{D}$ with $\omega_{t}(0)=0$. We define (see [Belinschi and Bercovici 2005]) $\mu^{\bigotimes t}$ by the relation

$$
\eta_{\mu}{ }^{\otimes t}(z)=\eta_{\mu}\left(\omega_{t}(z)\right) .
$$

Then we see that $H_{t}^{\prime}(0)>0, \omega_{t}^{\prime}(0)>0$ and that

$$
m_{1}\left(\mu^{\bigotimes t}\right)=\eta_{\mu}^{\prime \otimes t}(0)>0 .
$$

For $t>0$, we also define $\mu^{凶 t}$ by the relation

$$
k_{\mu 凶 t}(z)=\exp (t u(z)) .
$$

For this choice of the Boolean convolution power, we have

$$
m_{1}\left(\mu^{(凶 t}\right)>0 .
$$

Definition 3.19. Given $\mu \in \mathcal{M}_{\mathrm{T}}^{*}$ such that $m_{1}(\mu)>0$, we define a family of maps $\left\{\mathbb{M}_{t}\right\}_{t \neq 0}$ by

$$
\mathbb{M}_{t}(\mu)=\left(\mu^{\bigotimes(t+1)}\right)^{\bigotimes 1 /(t+1)},
$$

where we choose $\mu^{\bigotimes(t+1)}$ and $\mathbb{M}_{t}(\mu)$ in a way such that they have positive means.

The next result is a special case of [Arizmendi and Hasebe 2013, Theorem 4.4].

Lemma 3.20. Given $\mu \in M_{\mathrm{T}}^{*}$ with $m_{1}(\mu)>0$, the following assertions are true.

(1) $\mathbb{M}_{t+s}(\mu)=\mathbb{M}_{t}\left(\mathbb{M}_{s}(\mu)\right)$ for all $t, s \geq 0$.

(2) $\mathbb{M}_{1}(\mu)=\mathbb{M}(\mu)$.

Proof of Theorem 1.1. We set

$$
u(z)=\frac{1}{2} \int_{\mathrm{T}} \frac{1+\xi z}{1-\xi z} d v(\xi) .
$$

By (3-7) and the assumption (1-2), we have

$$
\frac{z}{\eta_{\mu}(z)}=\Sigma_{\lambda}\left(\eta_{v}(z)\right)=\exp (u(z)) \text {. }
$$


By Proposition 3.18, we see that $m_{1}(\mu)>0$. We therefore can choose the multiplicative convolution power $\mu^{\bigotimes(t+1)}$ such that $m_{1}\left(\mu^{\bigotimes(t+1)}\right)>0$.

Let $\eta_{t}$ be the principal subordination function of $v \otimes \lambda_{t}$ with respect to $v$ and $\omega_{t+1}$ be the subordination function of $\mu^{\bigotimes(t+1)}$ with respect to $\mu$. Let $\rho_{t}, \sigma_{t} \in M_{\mathrm{T}}$ such that $\eta_{\rho_{t}}=\eta_{t}$ and $\eta_{\sigma_{t}}=\omega_{t+1}$.

By Corollary 3.13, (1-2) implies that $\Sigma_{\rho_{t}}(z)=\Sigma_{\lambda_{t}}\left(\eta_{\mu}(z)\right)=\exp (t u(z))$. From the choice of $\mu^{\bigotimes(t+1)}$, the function $H_{t+1}(z):=z \exp (t u(z))$ is the left inverse of $\omega_{t+1}$ such that $H_{t+1}\left(\omega_{t+1}(z)\right)=z$ for all $z \in \mathbb{D}$, which implies that

$$
\Sigma_{\sigma_{t}}(z)=\exp (t u(z))
$$

We thus obtain that $\rho_{t}=\sigma_{t}$ and $\eta_{t}=\omega_{t+1}$.

Replacing $z$ by $\eta_{t}$ in (1-2), we obtain

$$
\begin{aligned}
\Sigma_{\lambda}\left(\eta_{\nu \bowtie \lambda_{t}}(z)\right) & =\Sigma_{\lambda}\left(\eta_{v}\left(\left(\eta_{t}(z)\right)\right)\right)=\frac{\eta_{t}(z)}{\eta_{\mu}\left(\eta_{t}(z)\right)} \\
& =\frac{\omega_{t+1}(z)}{\eta_{\mu}\left(\omega_{t+1}(z)\right)}=\frac{\omega_{t+1}(z)}{\left.\eta_{\mu^{\bigotimes(t+1)}}(z)\right)}=\left(\frac{z}{\eta_{\mu^{\bigotimes(t+1)}}(z)}\right)^{1 / t+1} .
\end{aligned}
$$

On the other hand, by the definition of $\mathbb{M}_{t}$, we have

$$
\frac{z}{\eta_{\mathbb{M}_{t}(\mu)}(z)}=\frac{z}{\eta_{\left(\mu^{凶(t+1)}\right) 凶(1 /(t+1))}(z)}=\left(\frac{z}{\eta_{\mu^{凶(t+1)}}(z)}\right)^{1 / t+1},
$$

completing the proof of Theorem 1.1.

Some examples and applications. We start with the multiplicative analogues of examples studied in [Anshelevich 2010; 2012; Arizmendi and Hasebe 2013; Belinschi and Nica 2008b]. We define the set

$$
(\mathrm{A})=\left\{\mu \in M_{\mathrm{T}}^{*}: m_{1}(\mu)=e^{-1 / 2}\right\} .
$$

By (3-22), the set $M_{\mathrm{T}}$ is in one-to-one correspondence with the set (A) via the bijection $v \leftrightarrow \mu$, such that $\Sigma_{\lambda}\left(\eta_{v}(z)\right)=z / \eta_{\mu}(z)$.

Definition 3.21. The bijective map $\Lambda: M_{\mathrm{T}} \rightarrow(\mathrm{A})$ is defined by

$$
\Sigma_{\lambda}\left(\eta_{v}(z)\right)=\frac{z}{\eta_{\Lambda[v]}(z)} \quad \text { for all } v \in \mathcal{M}_{\mathrm{T}}
$$

Using the $\Lambda$ notation, Theorem 1.1 implies that

$$
\Lambda\left[v \otimes \lambda_{t}\right]=\mathbb{M}_{t}[\Lambda(v)] \quad \text { for all } v \in \mathcal{M}_{\mathrm{T}} .
$$


Example 3.22. Let $\delta_{1}$ be the Dirac measure at 1 , and let $\mu=\Lambda\left[\delta_{1}\right]$, we have $z / \eta_{\mu}(z)=\Sigma_{\lambda}\left(\eta_{\delta_{1}}(z)\right)=\exp \left(\frac{1}{2}(1+z) /(1-z)\right)$. For $t \geq 0$, Theorem 1.1 implies that

$$
\frac{z}{\eta_{\mathbb{M}_{t}(\mu)}(z)}=\Sigma_{\lambda}\left(\eta_{\delta_{1} \otimes \lambda_{t}}(z)\right)=\Sigma_{\lambda}\left(\eta_{\lambda_{t}}(z)\right)
$$

In particular, when $t=1$,

$$
\eta_{\mathbb{M}_{1}(\mu)}(z)=\frac{z}{\Sigma_{\lambda}\left(\eta_{\lambda_{1}}(z)\right)}=\eta_{\lambda_{1}}(z),
$$

where we used the equality $\left(\left(z \Sigma_{\lambda}\right) \circ \eta_{\lambda}\right)(z)=z$ and $\lambda=\lambda_{1}$. Therefore, $\mathbb{M}_{1}(\mu)$ is the free multiplicative analogue of the normal distribution on $\mathrm{T}$.

Example 3.23. More generally, we consider $\lambda_{b, t}=\delta_{b} \otimes \lambda_{t}$ and $\mu_{b, t}=\Lambda\left[\lambda_{b, t}\right]$. Then

$$
\Sigma_{\lambda}\left(\eta_{\lambda_{b, t}}(z)\right)=\frac{z}{\eta_{\mu_{b, t}}(z)} \quad \text { for } t \neq 0 .
$$

On the other hand, Theorem 1.1 implies that

$$
\Sigma_{\lambda}\left(\eta_{\lambda_{b, t_{1}+t_{2}}}(z)\right)=\Sigma_{\lambda}\left(\eta_{\lambda_{b, t_{1}} \otimes \lambda_{2}}(z)\right)=\frac{z}{\eta_{\mathbb{M}_{t_{2}}\left(\mu_{b, t_{1}}\right)}(z)} \quad \text { for } t_{1}, t_{2} \geq 0,
$$

which yields that $\mu_{b, t_{1}+t_{2}}=\mathbb{M}_{t_{2}}\left(\mu_{b, t_{1}}\right)$ for $t_{1}, t_{2} \geq 0$.

We would like to provide another example which covers part of [Arizmendi and Hasebe 2013, Example 4.10].

Example 3.24. Let $P_{0}$ be the Haar measure on $\mathrm{T}$. Then by the free independence, $P_{0} \otimes \lambda_{t}=P_{0}$. We set $\mu=\Lambda\left[P_{0}\right]$, and we have

$$
\Sigma_{\lambda}\left(\eta_{P_{0} \otimes \lambda_{t}}(z)\right)=\Sigma_{\lambda}\left(\eta_{P_{0}}(z)\right)=\frac{z}{\eta_{\mu}(z)},
$$

which implies that $\mathbb{M}_{t}(\mu)=\mu$ for all $t \geq 0$. To calculate the distribution of $\mu$, we note that $\eta_{P_{0}} \equiv 0$, which shows that $\eta_{\mu}=e^{-1} z$, and thus $\psi_{\mu}(z)=z / e-z$. Using the identity

$$
\frac{1}{\pi}\left(\psi_{\mu}(z)+\frac{1}{2}\right)=\frac{1}{2 \pi} \int_{0}^{2 \pi} \frac{e^{i t}+z}{e^{i t}-z} d \mu\left(e^{-i t}\right),
$$

and Stieltjes's inversion formula, we obtain

$$
\mu(d t)=\frac{1}{2 \pi} \frac{1-e^{-2}}{1+e^{-2}-2 e^{-1} \cos (t)} d t, \quad 0 \leq t \leq 2 \pi .
$$

We then give some applications of results concerning infinity divisibility of the measures associated with subordination functions. For $\mu \in \mathcal{M}_{\mathrm{T}}$, we say $\mu$ is nontrivial if it is not a Dirac measure at a point on $\mathrm{T}$. 
Lemma 3.25. Given $\sigma \in \mathscr{I}(\bigotimes, \mathrm{T})$ which is non-trivial, and $0<\epsilon<1$, there exists a positive number $n(\epsilon)$ such that

$$
\eta_{\sigma_{t}}(\overline{\mathbb{D}}) \subset \mathbb{D}_{\epsilon}=\left\{z=r e^{i \theta}: 0 \leq r \leq \epsilon, 0 \leq \theta<2 \pi\right\}
$$

for any $t>n(\epsilon)$, where $\sigma_{t}=\sigma^{\otimes t}$.

Proof. If $\sigma=P_{0}$, the Haar measure on T, the result is trivial. If $\sigma \neq P_{0}$ is nontrivial, then by [Bercovici and Voiculescu 1992, Theorem 6.7], there exists a finite positive measure $v$ on $\mathrm{T}$ with $v(\mathrm{~T})>0, \alpha \in \mathbb{R}$, and an analytic function $u$ defined by

$$
u(z)=i \alpha+\int_{\mathrm{T}} \frac{1+\xi z}{1-\xi z} d v(\xi) \text { for } z \in \mathbb{D}
$$

such that $\Sigma_{\sigma}(z)=\exp (u(z))$. We choose $\sigma_{t} \in M_{\mathrm{T}}$ satisfying $\Sigma_{\sigma_{t}}(z)=\exp (t u(z))$. Noticing that other choices of the multiplicative free convolution power of $\sigma$ can be obtained from $\sigma_{t}$ by a rotation of a multiple of $2 \pi t$, it is enough to prove the assertion for $\sigma_{t}$.

We set $\Phi_{\sigma_{t}}=z \Sigma_{\sigma_{t}}(z)$; then, by Lemma 3.2, we have

$$
\Phi_{\sigma_{t}}^{-1}(\mathbb{D})=\eta_{\sigma_{t}}(\mathbb{D}) .
$$

For $z=r e^{i \theta} \in \mathbb{D}$, we calculate

$$
\begin{aligned}
\left|\Phi_{\sigma_{t}}(z)\right| & =r \exp \left(t \int_{\mathrm{T}} \frac{1-r^{2}}{|1-\xi z|^{2}} d v(\xi)\right) \\
& \geq r \exp \left(t \int_{\mathrm{T}} \frac{1-r^{2}}{|1+r|^{2}} d \nu(\xi)\right)=r \exp \left(t \cdot v(\mathrm{~T}) \frac{1-r}{1+r}\right) .
\end{aligned}
$$

Since

$$
\lim _{t \rightarrow \infty} r \exp \left(t \cdot v(\mathrm{~T}) \frac{1-r}{(1+r)}\right)=\infty
$$

we deduce that for any $0<\epsilon<1$, there exists a positive number $n(\epsilon)$ such that, for all $t>n(\epsilon)$, we have

$$
\left|\Phi_{\sigma_{t}}(z)\right|>1 \text { for }|z|=\epsilon .
$$

By Lemma 3.2, $\Phi_{\sigma_{t}}(\mathbb{D})$ is a simply connected domain which contains zero, which implies that

$$
\eta_{\sigma_{t}}(\mathbb{D})=\Phi_{\sigma_{t}}^{-1}(\mathbb{D}) \subset \mathbb{D}_{\epsilon}, \text { for } t>n(\epsilon) .
$$

The assertion follows because $\eta_{\sigma_{t}}$ extends to a continuous function on $\overline{\mathbb{D}}$.

For $\mu \in \mathcal{M}_{\mathrm{T}}$, we have

$$
\frac{1}{2 \pi}\left(\frac{1+\eta_{\mu}(z)}{1-\eta_{\mu}(z)}\right)=\frac{1}{2 \pi} \int_{0}^{2 \pi} \frac{e^{i \theta}+z}{e^{i \theta}-z} d \mu\left(e^{-i \theta}\right), \quad z \in \mathbb{D} .
$$


The real part of this function is the Poisson integral of the measure $d \mu\left(e^{-i \theta}\right)$, we can recover $\mu$ by Stieltjes's inversion formula. The functions

$$
\frac{1}{2 \pi} \Re\left(\frac{1+\eta_{\mu}\left(r e^{i \theta}\right)}{1-\eta_{\mu}\left(r e^{i \theta}\right)}\right)=\frac{1}{2 \pi} \frac{1-\left|\eta_{\mu}\left(r e^{i \theta}\right)\right|^{2}}{\left|1-\eta_{\mu}\left(r e^{i \theta}\right)\right|^{2}}
$$

converge to the density of $\mu\left(e^{-i \theta}\right)$ a.e. relative to Lebesgue measure, and they converge to infinity a.e. relative to the singular part of this measure.

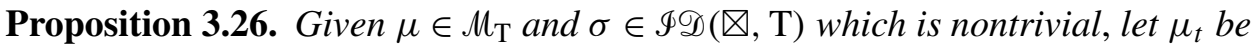
the unique probability measure on $\mathrm{T}$ such that

$$
\eta_{\mu_{t}}(z)=\eta_{\mu}\left(\eta_{\sigma_{t}}(z)\right)
$$

Then we have

$$
\lim _{t \rightarrow \infty} \sup _{\theta \in[0,2 \pi]}\left|\frac{d \mu_{t}\left(e^{i \theta}\right)}{d \theta}-\frac{1}{2 \pi}\right|=0,
$$

where $d \mu_{t}\left(e^{i \theta}\right) / d \theta$ is the density function of $\mu_{t}$ at $e^{i \theta}$ with respect to Lebesgue measure.

Proof. Given $0<\epsilon<1$, by Lemma 3.25, there exists $n(\epsilon)>0$ such that $\eta_{\sigma_{t}}\left(e^{i \theta}\right)<\epsilon$ for $t \geq n(\epsilon)$, which yields that $\eta_{\mu_{t}}(z)$ extends continuously to $\overline{\mathbb{D}}$. Thus

$$
\left|\eta_{\mu_{t}}\left(e^{i \theta}\right)\right|=\left|\eta_{\mu}\left(\eta_{\sigma_{t}}\left(e^{i \theta}\right)\right)\right| \leq\left|\eta_{\sigma_{t}}\left(e^{i \theta}\right)\right|<\epsilon,
$$

which implies that

$$
\frac{1-\epsilon}{1+\epsilon}=\frac{1-\epsilon^{2}}{|1+\epsilon|^{2}} \leq \frac{1-\left|\eta_{\mu_{t}}\left(e^{i \theta}\right)\right|^{2}}{\left|1-\eta_{\mu_{t}}\left(e^{i \theta}\right)\right|^{2}} \leq \frac{1}{|1-\epsilon|^{2}} .
$$

Since $\epsilon$ is arbitrary, combining (3-32) with (3-33), we prove our assertion.

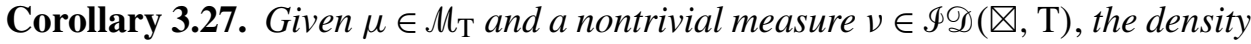
functions of the measures $\mu \otimes v_{t}$ converge to $1 / 2 \pi$ uniformly as $t \rightarrow \infty$; if $\mu \in M_{\mathrm{T}}$ is nontrivial, the density functions of the measures $\mu^{\bigotimes t}$ converge to $1 / 2 \pi$ uniformly as $t \rightarrow \infty$.

Proof. Noticing Corollary 3.13, Propositions 3.14, 3.26 and Subsection 3.3, we only need to prove the case of $\mu^{\bigotimes t}$ for $\mu \notin \mathcal{M}_{\mathrm{T}}^{*}$. We point out that the measures are nontrivial imply that the subordination distributions involved are nontrivial.

For $\mu \in M_{\mathrm{T}} \backslash M_{*}$, we have $\mu^{\otimes n}=P_{0}$, where $P_{0}$ is the Haar measure on T. Thus the assertion is true for this case. For $\mu \in \mathcal{M}_{\mathrm{T}} \cap \mathcal{M}_{*}$, but $\mu \notin \mathcal{M}_{\mathrm{T}}^{*}$, it is shown in [Belinschi and Bercovici 2005] that $\mu \otimes \mu \in \mathcal{M}_{\mathrm{T}}^{*}$; thus this case reduces to the case when $\mu \in M_{\mathrm{T}}^{*}$. This finishes the proof. 


\section{Multiplicative convolution on $M_{\mathbb{R}^{+}}$}

Multiplicative free convolution on $M_{\mathbb{R}^{+}}$. We are interested in the probability measures on the positive real line $\mathbb{R}^{+}$, which are different from the Dirac measure at zero, we thus set

$$
M_{\mathbb{R}^{+}}^{*}=M_{\mathbb{R}^{+}} \backslash\left\{\delta_{0}\right\}
$$

Given $\mu \in M_{\mathbb{R}^{+}}^{*}$, we define

$$
\psi_{\mu}(z)=\int_{0}^{+\infty} \frac{t z}{1-t z} d \mu(t)
$$

and $\eta_{\mu}(z)=\psi_{\mu}(z) /\left(1+\psi_{\mu}(z)\right)$. The transform $\eta_{\mu}$ is characterized by the following proposition; see [Belinschi and Bercovici 2005].

Proposition 4.1. Let $\eta: \mathbb{C} \backslash \mathbb{R}^{+} \rightarrow \mathbb{C}$ be an analytic function such that $\eta(\bar{z})=\overline{\eta(z)}$ for all $z \in \mathbb{C} \backslash \mathbb{R}^{+}$. Then the following two conditions are equivalent.

(1) $\eta=\eta_{\mu}$ for some $\mu \in \mathcal{M}_{\mathbb{R}^{+}}^{*}$.

(2) $\eta(0-)=0$ and $\arg (\eta(z)) \in[\arg z, \pi)$ for all $z \in \mathbb{C}^{+}$.

It can be shown that $\eta_{\mu}$ is invertible in some neighborhood of $(-\infty, 0)$, and we set $\Sigma_{\mu}(z)=\eta_{\mu}^{-1}(z) / z$ where $\eta_{\mu}^{-1}$ is defined in some neighborhood of $(\alpha, 0)$. Given two measures $\mu, v \in M_{\mathbb{R}^{+}}^{*}$, the multiplicative free convolution of $\mu$ and $v$ is the probability measure $\mu \otimes v$ in $\mu_{\mathbb{R}^{+}}^{*}$ such that

$$
\Sigma_{\mu \bowtie v}(z)=\Sigma_{\mu}(z) \Sigma_{v}(z)
$$

in some neighborhood of $(\alpha, 0)$, where these functions are defined.

It is known from [Belinschi and Bercovici 2007; Biane 1998] that there exist two analytic functions $\omega_{1}, \omega_{2}: \mathbb{C} \backslash \mathbb{R}^{+} \rightarrow \mathbb{C} \backslash \mathbb{R}^{+}$such that

(1) $\omega_{j}(0-)=0$ for $j=1,2$,

(2) for any $\lambda \in \mathbb{C}^{+}$, we have $\omega_{j}(\bar{\lambda})=\overline{\omega_{j}(\lambda)}$ for $j=1,2$,

(3) $\eta_{\mu 凶 v}(z)=\eta_{\mu}\left(\omega_{1}(z)\right)=\eta_{\nu}\left(\omega_{2}(z)\right)$ for $z \in \mathbb{C} \backslash \mathbb{R}^{+}$.

For simplicity, we say that $\omega_{1}$ (resp. $\omega_{2}$ ) is the subordination function of $\mu \nabla v$ with respect to $\mu$ (resp. $v$ ), and $\mu \nabla v$ is subordinated to $\mu$ and $v$.

The analogy of the Lévy-Khintchine in this setting was proved in [Bercovici and Voiculescu 1992; 1993]. A measure $\mu \in \mathcal{M}_{\mathbb{R}^{+}}$is $\otimes$-infinitely divisible if and only if $\Sigma_{\mu}(z)=\exp (u(z))$, with

$$
u(z)=a-b z+\int_{0}^{+\infty} \frac{1+t z}{z-t} d \sigma(t),
$$

where $b \in \mathbb{R}$ and $\sigma$ is a finite positive measure on $\mathbb{R}^{+}$. The analogue of the normal distribution in this context is given by $\Sigma_{\lambda_{t}}(z)=\exp ((t / 2)(z+1) /(z-1))$. 
Lemma 4.2. If $\mu, v \in \mathcal{M}_{\mathbb{R}^{+}}^{*}$, we have

$$
\eta_{\mu}(z)=\eta_{\mu \bowtie v}\left(z \Sigma_{v}\left(\eta_{\mu}(z)\right)\right)
$$

in some neighborhood of interval $(\alpha, 0)$.

The proof of Lemma 4.2 is identical to the proof of Lemma 3.3, therefore we omit the details.

For any $t>0$, assume that $\eta_{t}: \mathbb{D} \rightarrow \mathbb{D}$ is the subordination function of $\mu \nabla \lambda_{t}$ with respect to $\mu$, by Lemma 4.2 and the characterization of $\eta$-transform, there exists a probability measure $\rho_{t}$ in $\mathcal{M}_{\mathbb{R}^{+}}^{*}$ such that $\eta_{\rho_{t}}(z)=\eta_{t}(z)$. The argument in the proof of Lemma 3.4 implies the following result.

Proposition 4.3. The measure $\rho_{t}$ is $\bigotimes$-infinitely divisible and its $\Sigma$-transform is $\Sigma_{\rho_{t}}(z)=\Sigma_{\lambda_{t}}\left(\eta_{\mu}(z)\right)$, and

$$
\Sigma_{\lambda_{t}}\left(\eta_{\mu}(z)\right)=\exp \left(\frac{t}{2} \int_{0}^{+\infty} \frac{1+\xi z}{\xi z-1} d \mu(\xi)\right) .
$$

We now discuss free convolution semigroups. Given $t>1$, it is proved in [Belinschi and Bercovici 2005] that one can define $\mu^{\otimes t} \in \mathcal{M}_{\mathbb{R}^{+}}^{*}$ such that $\Sigma_{\mu^{\otimes t}}(z)=$ $\left(\Sigma_{\mu}(z)\right)^{t}$ for $z<0$ sufficiently close to zero. Similar to the case of $\mu_{\mathrm{T}}, \mu^{\otimes t}$ is subordinated with respect to $\mu$ and we denote the subordination function by $\omega_{t}$. By [Belinschi and Bercovici 2005, Theorem 2.6] and the characterization of $\eta$ transform, there exists a probability $\sigma_{t} \in \mathcal{M}_{\mathbb{R}^{+}}^{*}$ such that $\eta_{\sigma_{t}}(z)=\omega_{t+1}$ for all $t>0$. Moreover, $\sigma_{t}$ is $\otimes$-infinitely divisible and its $\Sigma$-transform is $\Sigma_{\sigma_{t}}(z)=\left[z / \eta_{\mu}(z)\right]^{t}$.

Multiplicative Boolean convolution on $\mathcal{M}_{\mathbb{R}^{+}}$and the semigroup $\mathbb{M}_{t}$. Bercovici [2006] proved that the multiplicative Boolean convolution does not preserve $\mathcal{M}_{\mathbb{B}^{+}}$. But we can still define $\mu^{凶 t}$ for $\mu \in \mathcal{M}_{\mathbb{R}^{+}}$and $0 \leq t \leq 1$ as follows. Let $k_{\mu}(z)=$ $z / \eta_{\mu}(z)$, the Boolean convolution power $\mu^{(\Downarrow t}$ is defined by

$$
k_{\mu \otimes t}(z)=\left(k_{\mu}(z)\right)^{t} .
$$

Definition 4.4 [Arizmendi and Hasebe 2013]. A family of maps from $\mathcal{M}_{\mathbb{R}^{+}}$to itself is defined by

$$
\mathbb{M}_{t}(\mu)=\left(\mu^{\bigotimes(t+1)}\right)^{\bigotimes(1 /(t+1))} .
$$

It is also shown in [Arizmendi and Hasebe 2013] that $\mathbb{M}_{t+s}=\mathbb{M}_{t} \circ \mathbb{M}_{s}$ for $t, s \geq 0$.

Analogous equations. Given a pair of probability measures $v, \mu \in \mathcal{M}_{\mathbb{R}^{+}}$, we also consider, as in the case $\mu_{\mathrm{T}}$, the semigroups $v \otimes \lambda_{t}$ and $\mu^{\bigotimes(t+1)}$, the subordination functions $\eta_{t}$ and $\omega_{t+1}$, and their associated probability measures $\rho_{t}, \sigma_{t}$ for all $t>0$. Since $\Sigma_{\rho_{t}}(z)=\Sigma_{\lambda_{t}}\left(\eta_{v}(z)\right)$ and $\Sigma_{\sigma_{t}}(z)=\left[z / \eta_{\mu}(z)\right]^{t}$, we deduce that $\eta_{t}=\omega_{t+1}$ if and only if

$$
\Sigma_{\lambda}\left(\eta_{\nu}(z)\right)=\frac{z}{\eta_{\mu}(z)}
$$


Applying the same argument as in the proof of the Theorem 1.1, we obtain the following result.

Theorem 4.5. Given a pair of probability measures $\mu, v \in \mathcal{M}_{\mathbb{R}^{+}}$such that

$$
\Sigma_{\lambda}\left(\eta_{\nu}(z)\right)=\frac{z}{\eta_{\mu}(z)}, \quad z \in \mathbb{C}^{+},
$$

we have $\Sigma_{\lambda}\left(\eta_{\nu \otimes \lambda_{t}}(z)\right)=\frac{z}{\eta_{\mathbb{M}_{t}(\mu)}(z)}, z \in \mathbb{C}^{+}$.

\section{A description of the analogue of the normal distribution}

Biane [1997a; 1997c] studied free Brownian motion and proved many important results. In this section, we give a new proof for the density functions of the free multiplicative analogue of the normal distributions, which was first obtained in [Biane 1997c] (See also [Demni and Hmidi 2012] for a different approach). Some results are new. For example, we show that $\lambda_{t}$ is unimodal for the circle case; and we show that $\Phi_{\lambda}^{-1}\left(\mathbb{C}^{+}\right)$contains infinitely many connected components where $\lambda$ is the free multiplicative analogue of the normal distribution on the positive half line with $\Sigma_{\lambda}(z)=\exp ((z+1) /(z-1))$. We also give a description of the boundaries $\Omega_{t}, \Omega$ (defined below), we observe that $\partial \Omega_{t}$ can be parametrized by $\theta$ and $\partial \Omega$ can be parametrized by $r$.

The circle case. Let $\lambda_{t} \in M_{\mathrm{T}}$ be the analogue of the normal distribution such that $\Sigma_{\lambda_{t}}(z)=\exp ((t / 2)(1+z) /(1-z))$. We set $\Phi_{t}(z)=z \Sigma_{\lambda_{t}}(z)$, and let $\Omega_{t}=\{z \in \mathbb{D}$ : $\left|\Phi_{t}(z)\right|<1$ \}. By Lemma 3.2, $\eta_{\lambda_{t}}$ extends continuously to the unit circle T, $\Omega_{t}$ is simply connected and bounded by a simple closed curve, and we have $\partial \Omega_{t}=\eta_{\lambda_{t}}(\mathrm{~T})$.

Observe that for $t \neq 4, \Phi_{t}$ has zeros of order one at $z_{1}(t)=\left(2-t+\sqrt{t^{2}-4 t}\right) / 2$ and $z_{2}(t)=\left(2-t-\sqrt{t^{2}-4 t}\right) / 2 . \Phi_{4}$ has a zero of order two at -1 ; and for all $t, \Phi_{t}$ has an essential singularity at 1 , and no other zeros and singularities. For $0<t<4$, $z_{1}(t), z_{2}(t) \in \mathrm{T}$ and $z_{2}(t)=\overline{z_{1}(t)}$, we let $\theta_{1}(t) \in(0, \pi)$ and $\theta_{2}(t) \in(\pi, 2 \pi)$ such that $z_{1}(t)=e^{i \theta_{1}(t)}$ and $z_{2}(t)=e^{i \theta_{2}(t)}$. We have $z_{1}(4)=z_{2}(4)=-1$ and for $t>4$, $z_{1}(t) \in(-1,0)$ and $z_{2}(t) \in(-\infty,-1)$.

We define

$$
g_{t}(r, \theta)=r \exp \left(\frac{t}{2} \frac{1-r^{2}}{1-2 r \cos \theta+r^{2}}\right)=\left|\Phi_{t}(z)\right|
$$

for $z=r e^{i \theta}$. The unit circle is parametrized by $\mathrm{T}=\left\{e^{i \theta}: 0 \leq \theta<2 \pi\right\}$.

Lemma 5.1. For $0<t<4, \partial \Omega_{t}=\left\{z=e^{i \theta}: \theta_{1}(t) \leq \theta \leq \theta_{2}(t)\right\} \cup \mathscr{L}_{1, t} \cup \mathscr{L}_{2, t}$, where $\mathscr{L}_{1, t}$ is an analytic curve, and $\mathscr{L}_{1, t}$ is in $\mathbb{D} \cap \mathbb{C}^{+}$except one of its endpoints, and $\mathscr{L}_{2, t}$ is the reflection of $\mathscr{L}_{1, t}$ about $x$-axis. $\mathscr{L}_{1, t}$ can be parametrized by $\gamma_{t}(u)(0 \leq u \leq 1)$ such that $\gamma_{t}(0) \in \mathbb{R}, \gamma_{t}(1)=z_{1}(t)$ and $\gamma_{t}(u) \subset \mathbb{D} \cap \mathbb{C}^{+}$for $0<u<1$. Moreover, $\left|\gamma_{t}(u)\right|$ is an increasing function of $u$ on the interval $[0,1]$. 
Proof. Observing that $\Phi_{t}(\bar{z})=\overline{\Phi_{t}(z)}$, we see that $\partial \Omega_{t}$ is symmetric with respect to $x$-axis. Since $\Omega_{t}$ is simply connected and $\partial \Omega_{t}$ is a simple closed curve, $\partial \Omega_{t}$ intersects $x$-axis at two points.

Restricting $\Phi_{t}$ to real numbers, we find that $\Phi_{t}(\mathbb{R}) \subset \mathbb{R}$, and that $\Phi_{t}$ is an increasing function on $(-1,1)$ since $\Phi_{t}^{\prime}(z)$ is positive for $z \in(-1,1)$. From $\Phi_{t}(-1)=-1$ and $\lim _{z \rightarrow 1^{-}} \Phi_{t}(z)=+\infty$, we deduce that

$$
\Phi_{t}^{-1}((-1,1))=(-1, x(t)),
$$

where $x(t)$ is the unique solution of the equation $\Phi_{t}(z)=1$ for $z \in(-1,1)$. The fact that $\Phi_{t}^{\prime}(z) \neq 0$ for $z \neq z_{t}(t), z_{2}(t)$ implies that $\Phi_{t}$ is locally invertible for $z \neq z_{1}(t), z_{2}(t)$. Combining the fact that $\Phi_{t}(\mathrm{~T} \backslash\{1\}) \subset \mathrm{T}$, we obtain that

$$
\left\{e^{i \theta}: \theta_{1}(t) \leq \theta \leq \theta_{2}(t)\right\} \subset \partial \Omega_{t}
$$

and $\partial \Omega_{t}$ has corners of opening $\pi / 2$ at $z_{1}(t)$ and $z_{2}(t)$.

Since $\Phi_{t}$ is a conformal mapping from $\Omega_{t}$ to $\mathbb{D}$, by the symmetry $\Phi_{t}(\bar{z})=\overline{\Phi_{t}(z)}$ and (5-1), noticing that $\Phi_{t}^{\prime}(0)=1$, we thus deduce that $\Phi_{t}\left(\Omega_{t} \cap \mathbb{C}^{+}\right) \subset \overline{\mathbb{D}} \cap \mathbb{C}^{+}$. Since $\partial \Omega_{t}$ is a simple closed curve, $z_{1}(t)$ and $x(t)$ are connected by $\partial \Omega_{t}$. It is clear that $\partial \Omega_{t} \backslash\left\{e^{i \theta}: \theta_{1}(t) \leq \theta \leq \theta_{2}(t)\right\}$ does not intersect with $\mathrm{T}$, we thus assume the curve $\gamma_{t}=\left\{\gamma_{t}(u): 0 \leq u \leq 1\right\}$ is the part of $\Omega_{t}$ which connects $z_{1}(t)$ and $x(t)$ such that $\gamma_{t}(0)=x(t), \gamma_{t}(1)=z_{1}(t)$ and $\gamma_{t}(u) \in \mathbb{D}$ for $0<u<1$.

We claim that $\left|\gamma_{t}(u)\right|$ is an increasing function of $u$ on the interval $[0,1]$. For given $0<r<1$, we define the function of $\theta$ by

$$
g_{t, r}(\theta)=g_{t}(r, \theta)=\left|\Phi_{t}\left(r e^{i \theta}\right)\right|
$$

Then $g_{t, r}$ is a strictly decreasing function of $\theta$ on the interval $[0, \pi]$. From the fact that $\Omega_{t}$ is simply connected, we deduce that, for $z_{0} \in \bar{\Omega}_{t} \cap \mathbb{D} \cap \mathbb{C}^{+}$, the arc

$$
\left\{r e^{i \theta}:|r|=\left|z_{0}\right|, \arg z_{0}<\theta \leq \pi\right\} \subset \Omega_{t} .
$$

Given $0<u_{1}<u_{2}<1$, we need to prove that $\left|\gamma_{t}\left(u_{1}\right)\right|<\left|\gamma_{t}\left(u_{2}\right)\right|$. Since $[0, x(t)] \subset$ $\overline{\Omega_{t}}$, we obtain from (5-2) that

$$
\left\{r e^{i \theta}: 0 \leq r \leq x(t), 0<\theta \leq \pi\right\} \subset \Omega_{t},
$$

which shows that $\left|\gamma_{t}\left(u_{1}\right)\right|>x(t)$. Suppose that $\left|\gamma_{t}\left(u_{1}\right)\right| \geq\left|\gamma_{t}\left(u_{2}\right)\right|$. There exists $0<u_{1}^{\prime} \leq u_{1}$ such that $\left|\gamma_{t}\left(u_{1}^{\prime}\right)\right|=\left|\gamma_{t}\left(u_{2}\right)\right|$. If $\arg \left(\gamma_{t}\left(u_{1}^{\prime}\right)\right)>\arg \left(\gamma_{t}\left(u_{2}\right)\right)$, then by $(5-2), \gamma_{t}\left(u_{2}\right) \in \Omega_{t}$ and thus $\gamma_{t}\left(u_{2}\right) \notin \partial \Omega_{t}$; if $\arg \left(\gamma_{t}\left(u_{1}^{\prime}\right)\right)<\arg \left(\gamma_{t}\left(u_{2}\right)\right)$, then $\gamma_{t}\left(u_{1}^{\prime}\right) \in \Omega_{t}$ and thus $\gamma_{t}\left(u_{1}^{\prime}\right) \notin \partial \Omega_{t}$. For both cases, we obtain a contradiction. Thus $\left|\gamma_{t}\left(u_{1}\right)\right|<\left|\gamma_{t}\left(u_{2}\right)\right|$ and our claim is proved. 
For $t>0$, we let $x_{1}(t) \in(0,1)$ be the unique solution of the equation $\Phi_{t}(z)=1$ for $z \in(0,1)$. For $0<t \leq 4$ we let $x_{2}(t)=-1$; for $t>4$, we let $x_{2}(t) \in(-1,0)$ be the unique solution of the equation $\Phi_{t}(z)=-1$ for $z \in(-1,0)$.

Lemma 5.2. For $t \geq 4, \partial \Omega_{t}=\mathscr{L}_{1, t} \cup \mathscr{L}_{2, t}$, where $\mathscr{L}_{1, t}$ is an analytic curve, and $\mathscr{L}_{1, t}$ is in $\mathbb{D} \cap \mathbb{C}^{+}$except its endpoints, and $\mathscr{L}_{2, t}$ is the reflection of $\mathscr{L}_{1, t}$ about $x$-axis. $\mathscr{L}_{1, t}$ can be parametrized by $\gamma_{t}(u)(0 \leq u \leq 1)$ such that $\gamma_{t}(0)=x_{1}(t), \gamma_{t}(1)=x_{2}(t)$ and $\gamma_{t}(u) \subset \mathbb{D} \cap \mathbb{C}^{+}$for $0<u<1$. Moreover, $\left|\gamma_{t}(u)\right|$ is an increasing function of $u$ on the interval $[0,1]$.

Proof. Recall that $\Phi_{4}$ has a zero of order two at -1 . For all $t>4, z_{2}(t)<-1$ and $z_{1} \in(-1,0)$. The assertion follows from the similar arguments in the proof Lemma 5.1.

From the proof of Lemmas 5.1 and 5.2, for $t>0$, we have $\Phi_{t}^{-1}((-1,1))=$ $\left(x_{2}(t), x_{1}(t)\right)$. Moreover, $x_{1}(t)=\min \left\{|z|: z \in \partial \Omega_{t}\right\}$ and $-x_{2}(t)=\max \left\{|z|: z \in \partial \Omega_{t}\right\}$.

Remark. In fact, for any $t>0$, from the equation

$$
g_{t}(r, \theta)=0, \quad 0<r<1,0 \leq \theta \leq \pi,
$$

we can prove that $d r / d \theta>0$ for $0<\theta<\pi$, which implies that if $z \in \partial \Omega_{t}$, the entire radius $\{r z: 0 \leq r<1\}$ is contained in $\Omega_{t}$. Therefore, $\partial \Omega_{t}$ can be parametrized by $\theta$.

Lemma 5.3. Using the same notations in Lemmas 5.1 and 5.2, for $t>0$, the function $\left|1-\gamma_{t}(u)\right|$ is an increasing function of $u$ on $[0,1]$.

Proof. We only prove the case when $0<t<4$, the proof for other cases are similar. Noticing that $\left|1-r e^{i \theta}\right|^{2}=1-2 \cos \theta+r^{2}$, since $\left|\gamma_{t}(u)\right|$ is an increasing function of $u$, to prove the assertion, we only need to prove that for the implicit function $r \exp \left((t / 2)\left(1-r^{2}\right) / h\right)=1$ of $r$ and $h$, the value of $h$ increases when $r$ increases on $(0,1)$. From this equation, we have $h=h(r)=-(t / 2)\left(1-r^{2}\right) /(\ln r)$. One can check that $h^{\prime}(r)>0$ for $0<r<1$, therefore $h$ is an increasing function of $r$.

Theorem 5.4. Denote by $A_{t}$ the support of $\lambda_{t}$.

(1) For $t>0$, the measure $\lambda_{t}$ has no singular part, and its density function is an analytic function. $A_{t_{1}} \subset A_{t_{2}}$ if $t_{1}<t_{2}<4 . A_{t} \subsetneq \mathrm{T}$ for $0<t<4$ and $A_{t}=\mathrm{T}$ for $t \geq 4$.

(2) The measure $\lambda_{t}$ is unimodal for all $t>0$ and its density is maximal at $z=1$ and is minimal at $z=-1$.

(3) The density fucntion $d \lambda_{t} / d \theta$ converges uniformly to $1 /(2 \pi)$ as $t \rightarrow \infty$.

Proof. Since $z=1$ is not in the closure of $\Omega_{t}=\eta_{\lambda_{t}}(\mathbb{D})$, the singular part of $\lambda_{t}$ vanishes. From the analyticity of $\Phi_{t}$ or a general theorem in [Belinschi and Bercovici 2005], the density function is analytic. 
For $0<t<4$, set $a_{1}(t)=\Phi_{t}\left(z_{1}(t)\right), a_{2}(t)=\Phi_{t}\left(z_{2}(t)\right)$. Note that $\eta_{\lambda_{t}}\left(\Phi_{t}(z)\right)=z$ for $z \in \bar{\Omega}_{t}$. From (3-32) we see that $A_{t}$ is the closed arc on $\mathrm{T}$ with endpoints $a_{1}(t), a_{2}(t)$ which contains 1 . Thus, to prove that $A_{t_{1}} \subset A_{t_{2}}$, it is enough to prove that $\arg \left(a_{1}(t)\right)$ is an increasing function of $t$. A direct computation shows that $\left|z_{1}(t)-1\right|^{2}=t$ and $\arg \left(\Sigma_{\lambda_{t}}\left(z_{1}(t)\right)\right)=\Im z_{1}(t)=\sqrt{t(4-t)} / 2$. We thus have

$$
\arg \left(a_{1}(t)\right)=\Im z_{1}(t)+\arg \left(z_{1}(t)\right)=\sin \left(\theta_{1}(t)\right)+\theta_{1}(t) .
$$

From $z_{1}(t)=\left(2-t+\sqrt{t^{2}-4 t}\right) / 2$ we see that $\theta_{1}(t)$ is an increasing function of $t$. The function $\theta \rightarrow \sin (\theta)+\theta$ is an increasing function on $(0, \pi)$. Thus $\arg \left(a_{1}(t)\right)$ is an increasing function of $t$ and (1) is proved.

To prove (2), recall that a probability measure is unimodal if its density with respect to Lebesgue measure has a unique local maximum. $\eta_{\lambda_{t}}$ extends continuously to $\mathrm{T}$, we thus have

$$
\frac{d \lambda_{t}\left(e^{-i \theta}\right)}{d \theta}=\frac{1}{2 \pi} \frac{1-\left|\eta_{\lambda_{t}}\left(e^{i \theta}\right)\right|^{2}}{\left|1-\eta_{\lambda_{t}}\left(e^{i \theta}\right)\right|^{2}}
$$

We first prove the case when $0<t<4$. From $\eta_{\lambda_{t}}\left(\Phi_{t}(z)\right)=z$ for $z \in \overline{\Omega_{t}}$ and $\eta_{\lambda_{t}}(1)=x(t)$, to prove $\lambda_{t}$ is unimodal, by the boundary correspondence, it is enough to show that the function $f_{t}$ of $u$ defined by

$$
f_{t}(u):=\frac{1-\left|\gamma_{t}(u)\right|^{2}}{\left|1-\gamma_{t}(u)\right|^{2}},
$$

is a decreasing function on $[0,1]$ and is maximal at 0 . Since $\gamma_{t}(u) \in \partial \Omega_{t}$, we have $\left|\Phi_{t}\left(\gamma_{t}(u)\right)\right|=1$. In other words, we have

$$
\left|\gamma_{t}(u)\right| \exp \left(\frac{t}{2} f_{t}(u)\right)=\left|\gamma_{t}(u)\right| \exp \left(\frac{t}{2} \frac{1-\left|\gamma_{t}(u)\right|^{2}}{\left|1-\gamma_{t}(u)\right|^{2}}\right)=1 .
$$

As we shown in Lemma 5.1 that the function $\left|\gamma_{t}(u)\right|$ is an increasing function of $u$, from (5-5), we deduce that $f_{t}$ is a decreasing function of $u$ and $\max \left\{f_{t}\right\}=f_{t}(0)$. By the symmetric property of the function $\Phi_{t}$ in Lemma 5.1, the density function is symmetric with respect to $x$-axis as well. Thus the density of $\lambda_{t}$ has only one local maximum at $\Phi_{t}\left(\gamma_{t}(0)\right)=\Phi_{t}\left(x_{1}(t)\right)=1$.

The proof for the case $t \geq 4$ is similar. In this case $A_{t}=\mathrm{T}$ and $\max \left\{f_{t}\right\}=f_{t}(0)$ and $\min \left\{f_{t}\right\}=f_{t}(1)$. Part (3) is a consequence of Corollary 3.27.

Remark. From the proof of Theorem 5.4, we see that, for $t<4$,

$$
\arg \left(a_{1}(t)\right)=\theta_{1}(t)+\sin \left(\theta_{1}(t)\right)=\frac{1}{2} \sqrt{t(4-t)}+\arccos \left(1-\frac{t}{2}\right),
$$

which implies a known result in [Biane 1997c], namely

$$
A_{t}=\left\{e^{i \theta}:-\frac{1}{2} \sqrt{t(4-t)}-\arccos \left(1-\frac{t}{2}\right) \leq \theta \leq \frac{1}{2} \sqrt{t(4-t)}+\arccos \left(1-\frac{t}{2}\right)\right\} .
$$


The positive half line case. Let $\lambda \in M_{\mathbb{R}^{+}}$be the analogue of the normal distribution such that $\Sigma_{\lambda}(z)=\exp ((z+1) /(z-1))$.

We restate [Bercovici and Voiculescu 1993, Proposition 6.14] in terms of $\eta$ and $\Sigma$ transforms as follows.

Lemma 5.5. Let $\mu$ be a $\bigotimes$-infinitely divisible measure on $\mathbb{R}^{+}$, and set $\Phi_{\mu}(z):=$ $z \Sigma_{\mu}(z)$.

(1) We have $\Phi_{\mu}\left(\eta_{\mu}(z)\right)=z$ for every $z \in \mathbb{C}^{+}$.

(2) The set $\left\{\eta_{\mu}(z): z \in \mathbb{C}^{+}\right\}=\Omega$, where $\Omega$ is the component of the set $\left\{z \in \mathbb{C}^{+}\right.$: $\left.\Im\left(\Phi_{\mu}(z)\right)>0\right\}$ whose boundary contains the left half line $(-\infty, 0)$. Moreover, $\eta_{\mu}\left(\Phi_{\mu}(z)\right)=z$ for $z \in \Omega$.

We set $\Phi_{\lambda}(z)=z \exp ((z+1) /(z-1))$. The following lemma is elementary.

Lemma 5.6. $\Phi_{\lambda}$ has zero of order one at $2-\sqrt{3}$ and $2+\sqrt{3}$, and $\Phi_{\lambda}$ has an essential singularity at 1 . These are the only zeros and singularities of $\Phi_{\lambda}$.

Theorem 5.7. The measure $\lambda$ has no singular part. The support of this measure is the closure of its interior, and this interior has only one connected component.

Proof. By [Bercovici and Voiculescu 1992, Theorem 7.5], the measure $\lambda$ has compact support on $\mathbb{R}^{+}$.

Let $\Omega$ be the component of $\left\{z \in \mathbb{C}^{+}: \Im\left(\Phi_{\lambda}(z)\right)>0\right\}$ whose boundary contains $(-\infty, 0)$. By Lemma $5.5, \eta_{\lambda}: \mathbb{C}^{+} \rightarrow \Omega$ is a conformal map and $\Phi_{\lambda}$ is its inverse map; thus $\Omega$ is simply connected. By Lemma $5.6, \partial \Omega$ is locally analytic. A general theorem in complex analysis tells us that $\eta_{\lambda}$ extends continuously to $\mathbb{C}^{+} \cup \mathbb{R}$ and it establishes a homeomorphism between the real axis and $\partial \Omega$. We continue to denote by $\eta_{\lambda}$ and $\Phi_{\lambda}$ their extensions.

We claim that

$$
\partial \Omega=(-\infty, 2-\sqrt{3}] \cup[2+\sqrt{3},+\infty) \cup \mathscr{L},
$$

where $\mathscr{L}$ is an analytic and open curve in $\mathbb{C}^{+}$with endpoints $2-\sqrt{3}$ and $2+\sqrt{3}$. We denote $\gamma(t)=\eta_{\lambda}(t), t \in \mathbb{R}$ be a parametrization of $\partial \Omega$. Set $t_{1}=\Phi_{\lambda}(2-\sqrt{3})>0$ and $t_{2}=\Phi_{\lambda}(2+\sqrt{3})>0$. Then $\eta_{\lambda}\left(t_{1}\right)=2-\sqrt{3}$ and $\eta_{\lambda}\left(t_{2}\right)=2+\sqrt{3}$, and $\mathscr{L}=\{\gamma(t)\}_{t_{1}<t<t_{2}}$. Note that

(1) $(-\infty, 0) \subset \partial \Omega$,

(2) $\Phi_{\lambda}^{\prime}(x)>0$ for all $x \in(-\infty, 2-\sqrt{3})$.

From this we deduce that $(-\infty, 2-\sqrt{3}) \subset \partial \Omega$. Lemma 5.6 tells us $\Phi_{\lambda}$ has a zero of order one at $2-\sqrt{3}$, therefore $\partial \Omega$ has a corner of opening $\pi / 2$ at $2-\sqrt{3}$. Note that $\Phi_{\lambda}^{\prime}(x)>0$ for all $x \in(2+\sqrt{3},+\infty)$, thus $(2+\sqrt{3},+\infty) \subset \partial \Omega$, and $\partial \Omega$ has a corner of opening $\pi / 2$ at $2+\sqrt{3}$. 
It remains to prove that $\mathscr{L} \cap \mathbb{R}=\varnothing$. First we show $1 \notin \mathscr{L}$. Suppose that is the case, and suppose $\gamma\left(t_{0}\right)=1$ where $t_{1}<t_{0}<t_{2}$, by continuity, we have

$$
\gamma(t) \exp \left(\frac{\gamma(t)+1}{\gamma(t)-1}\right)=\Phi_{\lambda}(\gamma(t))=\Phi_{\lambda}\left(\eta_{\lambda}(t)\right)=t
$$

for all $t \in \mathbb{R}$. Therefore in a small neighborhood of $t_{0}$, we have

$$
\frac{\gamma(t)+1}{\gamma(t)-1}=\ln \frac{t}{\gamma(t)} \text {. }
$$

The left side of the above equation blows up, while the right hand side is bounded. This contradiction tells us that $1 \notin \mathscr{L}$. Now suppose $\mathscr{L}$ touches the real axis at $x_{0} \in(2-\sqrt{3}, 1) \cup(1,2+\sqrt{3})$. Since $\Omega$ is connected, it is not hard to see that $x_{0}$ must be a critical point of $\Phi_{\lambda}$. This is not possible by Lemma 5.6. We therefore proved that $\mathscr{L} \subset \mathbb{C}^{+}$and the claim.

From the definitions of the Cauchy transform and $\eta$-transform, one can easily check that

$$
G_{\lambda}\left(\frac{1}{z}\right)=\frac{z}{1-\eta_{\lambda}(z)} .
$$

From the above equation we know that $G_{\lambda}$ extends to be a continuous function on $\mathbb{C} \cup \mathbb{R}$, and $\left\{x \in \mathbb{R}: \Im\left(G_{\mu}(x)\right)>0\right\}=\left(1 / t_{2}, 1 / t_{1}\right)$. By the Stieltjes inverse formula, we deduce that the support of $\lambda$ is $\left(1 / t_{2}, 1 / t_{1}\right)$. From the analyticity of the curve $\mathscr{L} \subset \mathbb{C}^{+}$, we conclude that $\lambda$ has positive and analytic density in the interior of its support.

We are interested in the level curves of the function

$$
f(r, \theta)=\theta-\frac{2 r \sin \theta}{1-2 r \cos \theta+r^{2}}=\arg \left(\Phi_{\lambda}(z)\right),
$$

where $z=r^{i \theta} \in \mathbb{C}^{+}$. For $t \leq 0$, set $\gamma_{t}=\left\{z=r e^{i \theta} \in \mathbb{C}^{+}: f(r, \theta)=t\right\}$.

Proposition 5.8. (A) $\gamma_{0}$ is a simple open curve with endpoints $2-\sqrt{3}, 2+\sqrt{3}$ and $\gamma_{0}=\mathscr{L}$.

(B) $\gamma_{t}$ is a simple open curve which starts at $z=1$ and ends at $z=1$ as well for all $t<0$.

Denote by $\Omega_{0}$ the open domain bounded $\gamma_{0} \cup[2-\sqrt{3}, 2+\sqrt{3}]$. For all $t<0$, denote by $\Omega_{t}$ the open domain bounded $\gamma_{t} \cup\{1\}$.

(C) For $t_{1}<t_{2} \leq 0$, we have $\Omega_{t_{1}} \subset \Omega_{t_{2}}$; and for all $t_{0} \leq 0, \Omega_{t_{0}}=\cup_{t<t_{0}} \Omega_{t}$.

Proof. Given $\theta \in(0, \pi)$, we define a function of $r$ by $f_{\theta}(r)=f(r, \theta)$ for $r \in(0,+\infty)$. We first note that $f(r, \theta)<\theta<\pi$ and observe that

$$
\lim _{r \rightarrow+\infty} f_{\theta}(r)=\theta
$$


Thus $\left\{z=r e^{i \theta}: f(r, \theta)>0,0<\theta<\pi\right\} \subset \Phi^{-1}\left(\mathbb{C}^{+}\right)$.

Given $\theta \in(0, \pi)$ and $t \leq 0$, the equation $f(r, \theta)=t$ is equivalent to the quadratic equation

$$
h_{\theta}(r):=(\theta-t) r^{2}-(2(\theta-t) \cos \theta+2 \sin \theta) r+\theta-t=0
$$

with discriminant $d(\theta, t)=[2(\theta-t) \cos \theta+2 \sin \theta]^{2}-4(\theta-t)^{2}$. We then rewrite $d(\theta, t)$ as follows.

$$
d(\theta, t)=4\left(1-\cos ^{2}(\theta)\right)\left[\frac{\sin \theta}{1+\cos \theta}+\theta-t\right] \cdot\left[\frac{\sin \theta}{1-\cos \theta}-\theta+t\right],
$$

The first two factors in (5-8) are never zero for $\theta \in(0, \pi)$; thus only the last factor in (5-8) matters to determine the sign of $d(\theta, t)$. We consider the function $k$ by $k(\theta)=\sin \theta /(1-\cos \theta)-\theta$ for $\theta \in(0, \pi)$, and calculate

$$
k^{\prime}(\theta)=\frac{1}{\cos \theta-1}-1<0,
$$

which implies that $k$ is a decreasing function of $\theta$. For $t \leq 0$, we now set $d_{t}(\theta):=$ $d(\theta, t)$. We then deduce that $d_{t}(\theta)=0$ has exactly one solution, which we denote by $\theta_{t}$, and $d_{t}(\theta)>0$ if and only if $0<\theta<\theta_{t}$. Therefore, the half line $r=\theta$ intersects with $\gamma_{t}$ at two points if and only if $0<\theta<\theta_{t}$ and the half line $r=\theta_{t}$ is tangent to $\gamma_{t}$. Moreover, $\theta_{t_{1}}<\theta_{t_{2}}$ if $t_{1}<t_{2} \leq 0$.

For the solutions of the equation $f(r, \theta)=0$, one can check as $\theta \rightarrow 0, r$ satisfying the equation $r^{2}-4 r+1$. Given $t<0$, for the solutions of the equation $f(r, \theta)=t$, we can easily see that $r$ tend to 1 as $\theta \rightarrow 0$. Now (A) and (B) follow from this observation.

Given $\theta \in(0, \pi)$, from (5-6), we see that the function $f_{\theta}(r)$ defined by $f_{\theta}(r)=$ $f(r, \theta)$ has exactly one local minimum at $r=1 . f_{\theta}(r)$ is a decreasing function of $r$ on $(0,1)$ and an increasing function of $r$ on $(1, \infty)$. Therefore, if the half line $r=\theta$ intersects $\gamma_{t}$ at two points, one of them is inside the unit circle of $\mathbb{C}$ and the other one is outside the unit circle. We conclude that $(\mathrm{C})$ is valid.

It is interesting to compare the next result with Proposition 2.2 and Lemma 3.2.

Corollary 5.9. We have $\Phi_{\lambda}^{-1}\left(\mathbb{C}^{+}\right)=\Omega \cup_{k=1}^{\infty}\left(\Omega_{(2 k-1) \pi} \backslash \Omega_{(2 k-2) \pi}\right)$. Moreover, $\Omega$ and $\Omega_{(2 k-1) \pi} \backslash \Omega_{(2 k-2) \pi}(k=1,2, \ldots)$ are all connected components of $\Phi_{\lambda}$. In particular, $\Phi_{\lambda}^{-1}\left(\mathbb{C}^{+}\right)$has infinitely many connected components.

We would like to point out that for $z=r e^{i \theta} \in \mathscr{L}=\gamma_{0}$, the curve $\mathscr{L}$ can be parametrized by $r$. Noticing (5-7) and (5-8), we first observe the following equivalences:

$$
d(\theta, 0)=0 \quad \Longleftrightarrow \quad \theta \cos \theta+\sin \theta=\theta \quad \Longleftrightarrow \quad r=1 .
$$


By (5-9), we see that (5-10) has exactly one solution $\theta_{0}$ for $\theta \in(0, \pi)$. By differentiating the equation $f(r, \theta)=0$, we obtain

$$
\frac{d \theta}{d r}=\frac{2 \theta \cos \theta+2 \sin \theta-2 \theta r}{r^{2}+2 \theta \sin \theta-4 \cos \theta+1} .
$$

Thus, $d \theta / d r=0$ if and only if $r=(\theta \cos \theta+\sin \theta) / \theta$. Fix $\theta$, the equation $f_{\theta}(r)=0$ is equivalent to the quadratic equation $\theta r^{2}-(2 \theta \cos \theta+2 \sin \theta) r+\theta=0$, from which we deduce that $r=(\theta \cos \theta+\sin \theta) / \theta$ if and only if $d(r, 0)=0$. From (5-11) and continuity of $d \theta / d r$, we see that $d \theta / d r>0$ for $0<\theta<\theta_{0}, r<1$ and $d \theta / d r<0$ for $0<\theta<\theta_{0}, r>1$. Therefore, for the solutions of the equation $f(r, \theta)=0, \theta$ is a function of $r$ and the curve $\mathscr{L}$ can be parametrized by $r$.

Denote by $g$ the density function of $\lambda$. From the equation $G_{\lambda}(1 / x)=x /\left(1-\eta_{\lambda}(x)\right)$, we obtain the following formula for the density function of $\lambda$.

Proposition 5.10. Given $z=r e^{i \theta} \in \gamma_{0}=\mathscr{L}$, we have

$$
g(1 / x)=\theta \Phi_{\lambda}(z)=r \theta \exp \left(\frac{r^{2}-1}{1-2 r \cos \theta+r^{2}}\right)
$$

where $x=\Phi_{\lambda}(z)$.

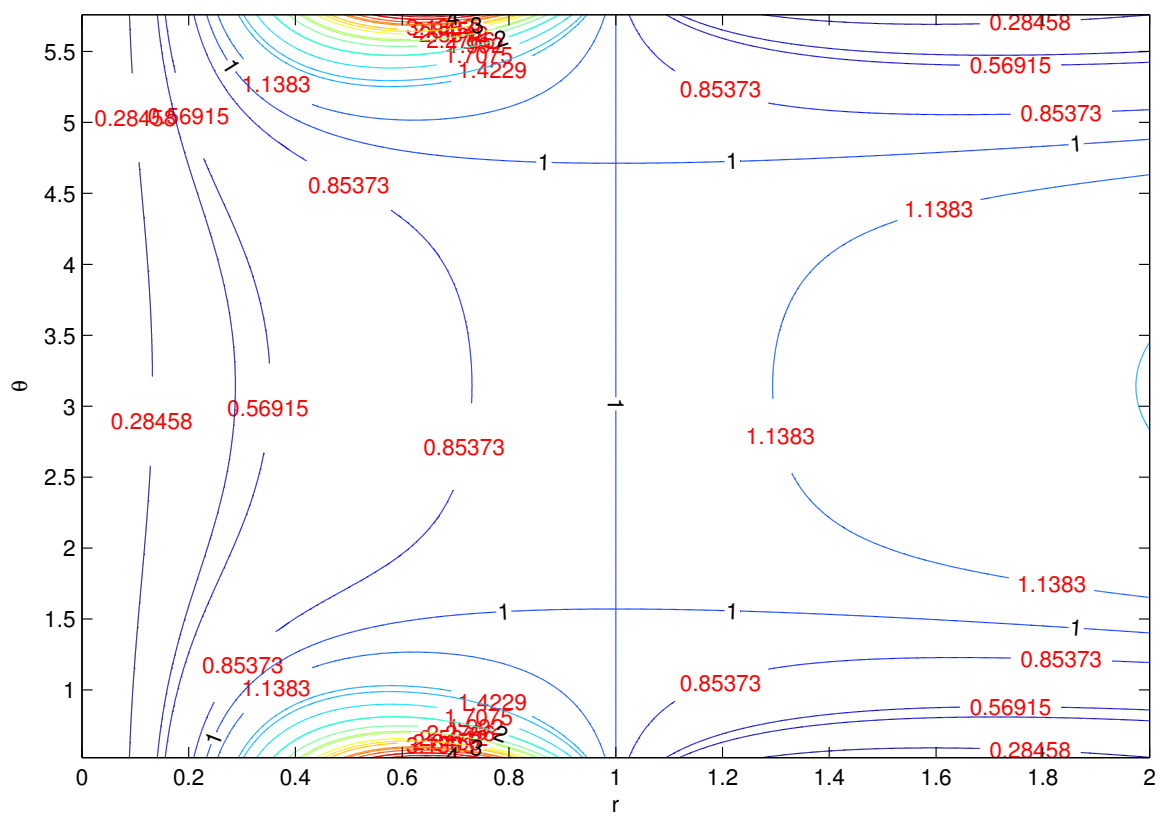

Figure 1. Level curves of $g_{2}(r, \theta)=\left|\Phi_{2}\left(r e^{i \theta}\right)\right|$. The vertical axis indicates $\theta$, and the horizontal axis indicates $r$. 


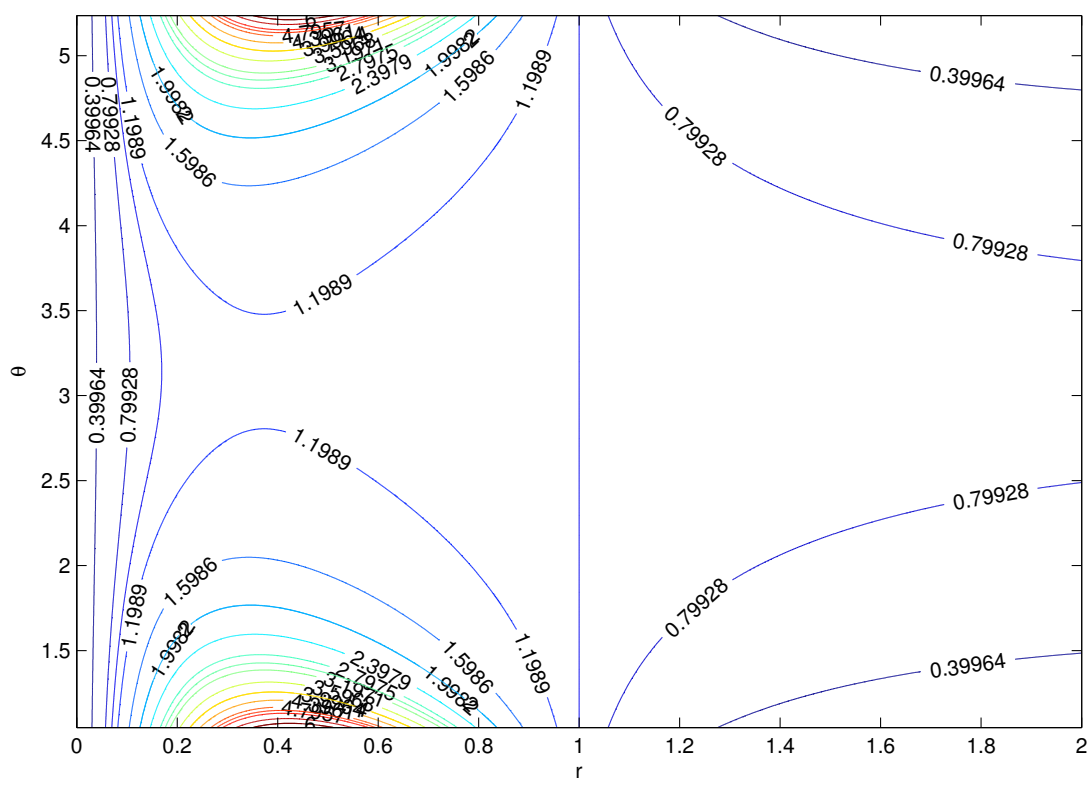

Figure 2. Level curves of $g_{5}(r, \theta)=\left|\Phi_{5}\left(r e^{i \theta}\right)\right|$. The vertical axis indicates $\theta$, and the horizontal axis indicates $r$.

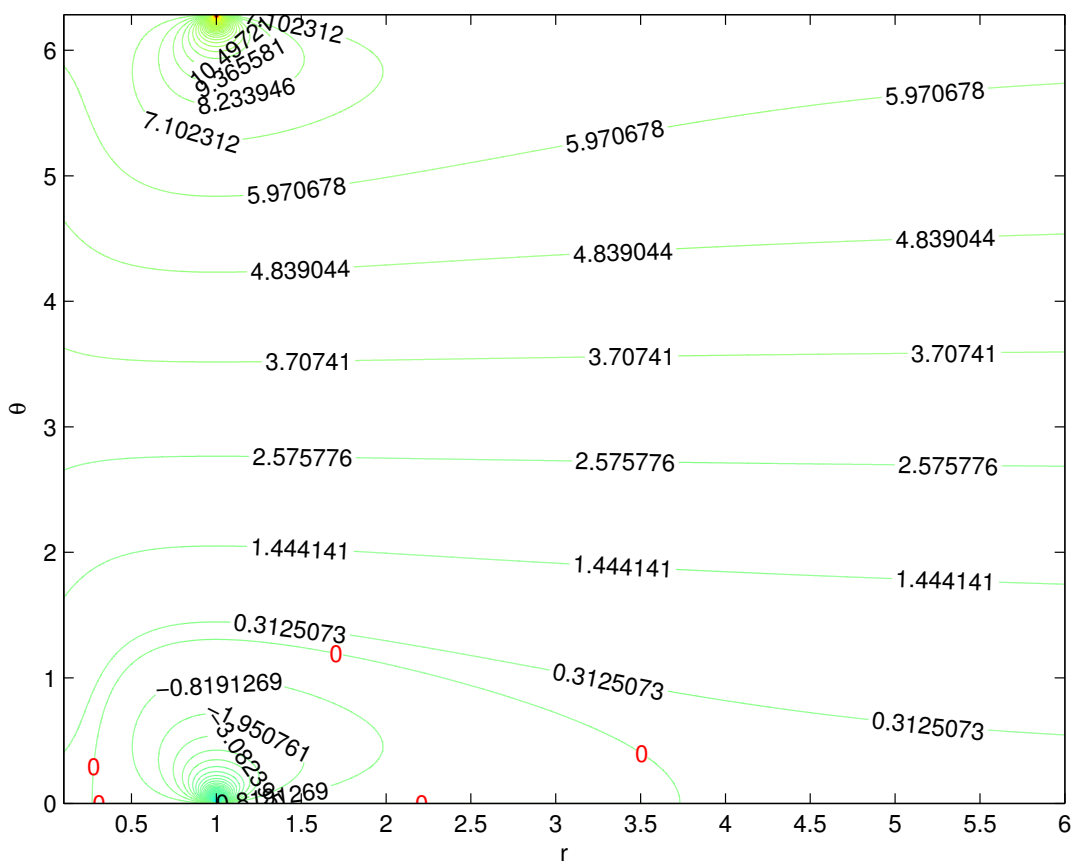

Figure 3. Level curves of $f(r, \theta)=\arg \left(\Phi_{\lambda}\left(r e^{i \theta}\right)\right)$. The vertical axis indicates $\theta$, and the horizontal axis indicates $r$. 


\section{Acknowledgements}

I am grateful to my advisor, Professor Hari Bercovici, for encouragement and many helpful discussions which made this work possible. I thank Octavio Arizmendi for informing me of [Arizmendi 2012] and Professor Michael Anshelevich for his interest. I would also like to thank Professor Eric Bedford for several helpful conversations and Aimin Huang for his careful reading of a preliminary version of this paper.

\section{References}

[Anshelevich 2010] M. Anshelevich, "Free evolution on algebras with two states", J. Reine Angew. Math. 638 (2010), 75-101. MR 2012d:46155 Zbl 1195.46069

[Anshelevich 2011a] M. Anshelevich, "Generators of some non-commutative stochastic process", preprint, 2011. arXiv 1104.1381v2

[Anshelevich 2011b] M. Anshelevich, “Two-state free Brownian motions”, J. Funct. Anal. 260:2 (2011), 541-565. MR 2012c:46147 Zbl 1221.46066

[Anshelevich 2012] M. Anshelevich, "Free evolution on algebras with two states II", preprint, 2012. arXiv $1204.0289 \mathrm{v} 1$

[Arizmendi 2012] O. Arizmendi, “ $k$-divisible random variables in free probability", preprint, 2012. arXiv 1203.4780v1

[Arizmendi and Hasebe 2013] O. Arizmendi and T. Hasebe, "Semigroups related to additive and multiplicative, free and Boolean convolutions", Studia Math. 215:2 (2013), 157-185. MR 3071490 Zbl 06172514 arXiv 1105.3344v3

[Belinschi and Bercovici 2004] S. T. Belinschi and H. Bercovici, "Atoms and regularity for measures in a partially defined free convolution semigroup", Math. Z. 248:4 (2004), 665-674. MR 2006i:46095 Zbl 1065.46045

[Belinschi and Bercovici 2005] S. T. Belinschi and H. Bercovici, "Partially defined semigroups relative to multiplicative free convolution”, Int. Math. Res. Not. 2005:2 (2005), 65-101. MR 2006f:46061 Zbl 1092.46046

[Belinschi and Bercovici 2007] S. T. Belinschi and H. Bercovici, "A new approach to subordination results in free probability", J. Anal. Math. 101 (2007), 357-365. MR 2008i:46059 Zbl 1142.46030

[Belinschi and Nica 2008a] S. T. Belinschi and A. Nica, " $\eta$-series and a Boolean Bercovici-Pata bijection for bounded $k$-tuples", Adv. Math. 217:1 (2008), 1-41. MR 2009c:46088 Zbl 1129.46052

[Belinschi and Nica 2008b] S. T. Belinschi and A. Nica, "On a remarkable semigroup of homomorphisms with respect to free multiplicative convolution", Indiana Univ. Math. J. 57:4 (2008), 1679-1713. MR 2009f:46087 Zbl 1165.46033

[Belinschi and Nica 2009] S. T. Belinschi and A. Nica, "Free Brownian motion and evolution towards $\boxplus$-infinite divisibility for $k$-tuples”, Internat. J. Math. 20:3 (2009), 309-338. MR 2010g:46108 Zbl 1173.46306

[Bercovici 2006] H. Bercovici, “On Boolean convolutions”, pp. 7-13 in Operator theory 20, edited by K. R. Davidson et al., Theta Ser. Adv. Math. 6, Theta, Bucharest, 2006. MR 2007m:46105 Zbl 1182.74031

[Bercovici and Pata 1999] H. Bercovici and V. Pata, "Stable laws and domains of attraction in free probability theory”, Ann. of Math. (2) 149:3 (1999), 1023-1060. MR 2000i:46061 Zbl 0945.46046 
[Bercovici and Voiculescu 1992] H. Bercovici and D.-V. Voiculescu, "Lévy-Hinčin type theorems for multiplicative and additive free convolution”, Pacific J. Math. 153:2 (1992), 217-248. MR 93k:46052 Zbl 0769.60013

[Bercovici and Voiculescu 1993] H. Bercovici and D.-V. Voiculescu, "Free convolution of measures with unbounded support", Indiana Univ. Math. J. 42:3 (1993), 733-773. MR 95c:46109 Zbl 0806.46070

[Biane 1997a] P. Biane, "Free Brownian motion, free stochastic calculus and random matrices", pp. 1-19 in Free probability theory (Waterloo, ON, 1995), edited by D.-V. Voiculescu, Fields Inst. Commun. 12, Amer. Math. Soc., Providence, RI, 1997. MR 97m:46104 Zbl 0873.60056

[Biane 1997b] P. Biane, "On the free convolution with a semi-circular distribution", Indiana Univ. Math. J. 46:3 (1997), 705-718. MR 99e:46084 Zbl 0904.46045

[Biane 1997c] P. Biane, "Segal-Bargmann transform, functional calculus on matrix spaces and the theory of semi-circular and circular systems", J. Funct. Anal. 144:1 (1997), 232-286. MR 97k:22011 Zbl 0889.47013

[Biane 1998] P. Biane, "Processes with free increments", Math. Z. 227:1 (1998), 143-174. MR 99e: 46085 Zbl 0902.60060

[Chistyakov and Götze 2011] G. P. Chistyakov and F. Götze, "The arithmetic of distributions in free probability theory”, Cent. Eur. J. Math. 9:5 (2011), 997-1050. MR 2012f:46136 Zbl 1239.46046

[Chistyakov and Götze 2013] G. P. Chistyakov and F. Götze, "Asymptotic expansions in the CLT in free probability”, Probab. Theory Related Fields 157:1-2 (2013), 107-156. MR 3101842 arXiv $1109.4844 \mathrm{v} 2$

[Demni and Hmidi 2012] N. Demni and T. Hmidi, "Spectral distribution of the free unitary Brownian motion: another approach", pp. 191-206 in Séminaire de Probabilités XLIV, edited by C. Donati-Martin et al., Lecture Notes in Math. 2046, Springer, Heidelberg, 2012. MR 2953348 Zbl 1257.46036 arXiv 1103.4693

[Franz 2008] U. Franz, "Boolean convolution of probability measures on the unit circle", pp. 83-94 in Analyse et probabilités, edited by P. Biane et al., Sémin. Congr. 16, Soc. Math. France, Paris, 2008. MR 2011c:46136 Zbl 1180.60011 arXiv math/0403243

[Hille 1959] E. Hille, Analytic function theory, vol. 1, Ginn, Boston, 1959. MR 21 \#6415 Zbl 0088. 05204

[Lenczewski 2007] R. Lenczewski, "Decompositions of the free additive convolution", J. Funct. Anal. 246:2 (2007), 330-365. MR 2008d:28009 Zbl 1129.46055

[Maassen 1992] H. Maassen, "Addition of freely independent random variables", J. Funct. Anal. 106:2 (1992), 409-438. MR 94g:46069 Zbl 0784.46047

[Nica 2009] A. Nica, "Multi-variable subordination distributions for free additive convolution", $J$. Funct. Anal. 257:2 (2009), 428-463. MR 2010j:46121 Zbl 1186.46068

[Nica and Speicher 1996] A. Nica and R. Speicher, "On the multiplication of free $N$-tuples of noncommutative random variables”, Amer. J. Math. 118:4 (1996), 799-837. MR 98i:46069 Zbl 0856.46035

[Rao and Speicher 2007] N. R. Rao and R. Speicher, "Multiplication of free random variables and the $S$-transform: the case of vanishing mean", Electron. Comm. Probab. 12 (2007), 248-258. MR 2008f:46082 Zbl 1133.46038

[Speicher and Woroudi 1997] R. Speicher and R. Woroudi, "Boolean convolution", pp. 267-279 in Free probability theory (Waterloo, ON, 1995), edited by D.-V. Voiculescu, Fields Inst. Commun. 12, Amer. Math. Soc., Providence, RI, 1997. MR 98b:46084 Zbl 0872.46033

[Voiculescu 1986] D.-V. Voiculescu, "Addition of certain noncommuting random variables", J. Funct. Anal. 66:3 (1986), 323-346. MR 87j:46122 Zbl 0651.46063 
[Wang 2008] J.-C. Wang, "Limit laws for Boolean convolutions", Pacific J. Math. 237:2 (2008), 349-371. MR 2009h:46128 Zbl 1152.46055

[Wang 2010] J.-C. Wang, "Local limit theorems in free probability theory", Ann. Probab. 38:4 (2010), 1492-1506. MR 2011i:46081 Zbl 1202.46078

Received October 13, 2012. Revised August 30, 2013.

PING ZHONG

DEPARTMENT OF MATHEMATICS

INDIANA UNIVERSITY BLOOMINGTON

RaWles HaLl, 831 East Third Street

BLOOMINGTON, IN 47405

UNITED STATES

Current address:

SCHOOL OF MATHEMATICS AND STATISTICS

WUHAN UNIVERSITY

WUHAN, HubEI 430072

CHINA

pzhong@indiana.edu 


\title{
PACIFIC JOURNAL OF MATHEMATICS
}

\author{
msp.org/pjm
}

Founded in 1951 by E. F. Beckenbach (1906-1982) and F. Wolf (1904-1989)

\section{EDITORS}

Don Blasius (Managing Editor)

Department of Mathematics

University of California

Los Angeles, CA 90095-1555

blasius@math.ucla.edu

\author{
Paul Balmer \\ Department of Mathematics \\ University of California \\ Los Angeles, CA 90095-1555 \\ balmer@math.ucla.edu \\ Robert Finn \\ Department of Mathematics \\ Stanford University \\ Stanford, CA 94305-2125 \\ finn@math.stanford.edu \\ Sorin Popa \\ Department of Mathematics \\ University of California \\ Los Angeles, CA 90095-1555 \\ popa@math.ucla.edu
}

\author{
Vyjayanthi Chari \\ Department of Mathematics \\ University of California \\ Riverside, CA 92521-0135 \\ chari@math.ucr.edu \\ Kefeng Liu \\ Department of Mathematics \\ University of California \\ Los Angeles, CA 90095-1555 \\ liu@math.ucla.edu \\ Jie Qing \\ Department of Mathematics \\ University of California \\ Santa Cruz, CA 95064 \\ qing@ cats.ucsc.edu
}

\section{PRODUCTION}

Silvio Levy, Scientific Editor, production@msp.org

\section{SUPPORTING INSTITUTIONS}

ACADEMIA SINICA, TAIPEI

CALIFORNIA INST. OF TECHNOLOGY

INST. DE MATEMÁTICA PURA E APLICADA

KEIO UNIVERSITY

MATH. SCIENCES RESEARCH INSTITUTE

NEW MEXICO STATE UNIV.

OREGON STATE UNIV.

\author{
STANFORD UNIVERSITY \\ UNIV. OF BRITISH COLUMBIA \\ UNIV. OF CALIFORNIA, BERKELEY \\ UNIV. OF CALIFORNIA, DAVIS \\ UNIV. OF CALIFORNIA, LOS ANGELES \\ UNIV. OF CALIFORNIA, RIVERSIDE \\ UNIV. OF CALIFORNIA, SAN DIEGO \\ UNIV. OF CALIF., SANTA BARBARA
}

\author{
Daryl Cooper \\ Department of Mathematics \\ University of California \\ Santa Barbara, CA 93106-3080 \\ cooper@math.ucsb.edu \\ Jiang-Hua Lu \\ Department of Mathematics \\ The University of Hong Kong \\ Pokfulam Rd., Hong Kong \\ jhlu@maths.hku.hk \\ Paul Yang \\ Department of Mathematics \\ Princeton University \\ Princeton NJ 08544-1000 \\ yang@math.princeton.edu
}

These supporting institutions contribute to the cost of publication of this Journal, but they are not owners or publishers and have no responsibility for its contents or policies.

See inside back cover or msp.org/pjm for submission instructions.

The subscription price for 2014 is US $\$ 410 /$ year for the electronic version, and \$535/year for print and electronic.

Subscriptions, requests for back issues and changes of subscribers address should be sent to Pacific Journal of Mathematics, P.O. Box 4163, Berkeley, CA 94704-0163, U.S.A. The Pacific Journal of Mathematics is indexed by Mathematical Reviews, Zentralblatt MATH, PASCAL CNRS Index, Referativnyi Zhurnal, Current Mathematical Publications and Web of Knowledge (Science Citation Index).

The Pacific Journal of Mathematics (ISSN 0030-8730) at the University of California, c/o Department of Mathematics, 798 Evans Hall \#3840, Berkeley, CA 94720-3840, is published twelve times a year. Periodical rate postage paid at Berkeley, CA 94704, and additional mailing offices. POSTMASTER: send address changes to Pacific Journal of Mathematics, P.O. Box 4163, Berkeley, CA 94704-0163.

PJM peer review and production are managed by EditFLOW ${ }^{\circledR}$ from Mathematical Sciences Publishers.

\section{PUBLISHED BY}

\section{mathematical sciences publishers \\ nonprofit scientific publishing}

http://msp.org/

(C) 2014 Mathematical Sciences Publishers 


\section{PACIFIC JOURNAL OF MATHEMATICS}

Volume 269 No. $1 \quad$ May 2014

The asymptotic behavior of Palais-Smale sequences on manifolds with $\quad 1$ boundary

SÉRGIO AlMARAZ

The cup subalgebra of a $\mathrm{II}_{1}$ factor given by a subfactor planar algebra is maximal amenable

ARNAUD BROTHIER

Representation theory of type B and C standard Levi $W$-algebras

JONATHAN BROWN and SIMON M. GOODWIN

New invariants for complex manifolds and rational singularities

RONG DU and YUN GAO

Homogeneity groups of ends of open 3-manifolds

DENNIS J. GARITY and DUŠAN REPOVŠ

On the concircular curvature of a $(\kappa, \mu, \nu)$-manifold

Florence Gouli-Andreou and Evaggelia Moutafi

Genuses of cluster quivers of finite mutation type

FANG LI, JICHUN LIU and YICHAO YANG

Taut foliations in knot complements

TAO LI and RACHEL ROBERTS

On the set of maximal nilpotent supports of supercuspidal representations 169

QIN YUJUN

The natural filtrations of finite-dimensional modular Lie superalgebras of 199 Witt and Hamiltonian type

Keli Zheng, Yongzheng ZHANG and WeI Song

Free Brownian motion and free convolution semigroups: multiplicative 219 case

PING ZHONG 